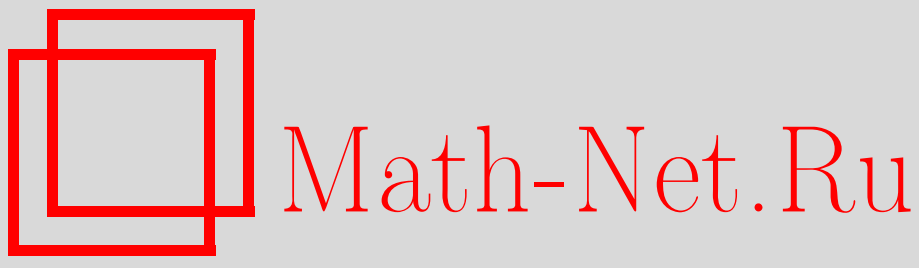

Е. А. Сатаев, Отсутствие устойчивых траекторий у неавтономных возмущений систем типа системы Лоренца, Матем. сб., 2005, том 196, номер 4, 99-134

DOI: https://doi.org/10.4213/sm1288

Использование Общероссийского математического портала Math-Net.Ru подразумевает, что вы прочитали и согласны с пользовательским соглашением

http: //www. mathnet.ru/rus/agreement

Параметры загрузки:

IP: 3.82 .47 .9

26 апреля 2023 г., 15:09:48 
УДК 517.938

\author{
Е. А. Сатаев
}

\title{
Отсутствие устойчивых траекторий у неавтономных возмущений систем типа системы Лоренца
}

\footnotetext{
Рассматриваются системы в некоторой ограниченной области пространства $\mathbb{R}^{3}$, которые являются обобщением системы Лоренца. Доказывается, что при вьполнении некоторых условий равномерной гиперболичности малые неавтономные возмущения не приводят к появлению устойчивых траекторий.

Библиография: 23 названия.
}

\section{§1. Введение}

Система уравнений Лоренца имеет вид

$$
\dot{x}=\sigma(y-x), \quad \dot{y}=r x-y-x z, \quad \dot{z}=-b z+x y .
$$

Эта система была введена в работе [1] как простейшая модель конвекции жидкости и исследована Е. Лоренцем с помощью численного счета в работе [2]. Лоренц обнаружил, что при значениях параметров $\sigma=10, r=28, b=\frac{8}{3}$ поведение траекторий уравнения подобно поведению реализаций случайного процесса. Естественно возникает вопрос: в чем причина такого поведения - в ошибках при аппроксимации решения дифференциального уравнения разностной схемой или во внутренних свойствах системы?

Внимание специалистов по динамическим системам к системе Лоренца было привлечено благодаря работам [3], [4], после чего ей было посвяшено огромное количество работ.

В большинстве математических работ изучается так называемая геометрическая модель системы Лоренца. Это модель отображения последования на плоскости $z=r-1$. Отображение является разрывным. Разрывы возникают на линии пересечения устойчивого многообразия неподвижной точки $O(0,0,0)$ и плоскости $z=r-1$. В модели постулируется, что для отображения последования выполнены условия гиперболичности (их формулировка приводится ниже).

Подобные отображения изучались во многих работах (см., например, [5]-[9] и др.). Достаточно хорошо исследована топологическая структура. Доказано, что для подобных отображений имеется естественная инвариантная мера типа Синая-Боуэна-Рюэлля (SBR-мера), относительно которой отображение имеет сильные стохастические свойства (убывание корреляций, центральная предельная теорема и др.) [10]-[13].

Работа вьполнена при поддержке РФФИ-Калуга (грант № 04-01-97204) и Программы поддержки ведущих научных школ РФ (грант № НШ-457.2003.01). 
Полное доказательство гиперболичности для реальной системы Лоренца с помощью компьютера было приведено недавно [14]. До этого разньми авторами проводились вычисления на компьютере, только подтверждающие гипотезу о равномерной гиперболичности (возможно, правильнее было бы сказать "не противоречащие гипотезе о гиперболичности"). Имеются другие системы лоренцева типа, для которых гиперболичность удалось доказать без применения компюютера (см. $[15],[16])$.

Изучались и возмушения системы Лоренца. Возмущение можно определить как систему

$$
\dot{x}=\sigma(y-x)+h_{1}, \quad \dot{y}=r x-y-x z+h_{2}, \quad \dot{z}=-b z+x y+h_{3},
$$

где $h_{1}, h_{2}, h_{3}$ - малые функции переменных $x, y, z$. Подобные возмущения мы будем называть автономными. Из-за разрывов отображение последования не является структурно устойчивым, поэтому и поток не является структурно устойчивьм. Однако многие свойства (в частности, гиперболическая структура) при возмущениях сохраняются.

Те свойства, которые при возмушениях сохраняются, называются грубыми. Свойства, которые не сохраняются при возмущениях, назьваются негрубъми. Краткий обзор грубых и негрубых свойств для систем типа системы Лоренца приведен в [17].

Неавтономное возмущение (или возмушение, зависящее от времени) - это система (2), в которой функции $h_{1}, h_{2}, h_{3}$ зависят от переменных $x, y, z$ и времени $t$.

Для неавтономных возмушений набор грубых свойств гораздо беднее, чем для автономных. В частности, гиперболическая структура при неавтономных возмущениях разрушается (подробнее см. пример в $\S 5$ ). По этой причине неавтономные возмушения потоков в гиперболической теории являются весьма слабо изученными.

В настоящей работе изучаются малые неавтономные возмущения систем типа системы Лоренца (точное определение см. ниже). Решается следующая

ЗАДАЧА. Может ли появиться устойчивая траектория у малого неавтономного возмущения системы типа системы Лоренща?

Гипотеза о том, что ответ отрицательный (по крайней мере для периодических возмущений), высказывалась Л.П. Шильниковым. Гипотеза основывалась примерно на следующих соображениях: у потока, определяемого системой Лоренца, имеется инвариантное одномерное сильно устойчивое слоение. Кроме того, в двумерном неправлении, трансверсальном сильно устойчивому слоению, имеется свойство экспоненциального увеличения площади. При периодическом возмушении рассматривается поток уже в четырехмерном пространстве. При этом одномерное сильно устойчивое слоение сохраняется, а растяжение двумерной площади трансформируется в увеличение трехмерного объема. Отсюда выводится, что периодические устойчивые траектории отсутствуют.

Ниже мы увидим, что ответ на вопрос отрицательњый даже для неавтономного возмущения. Мы докажем это для более общих систем, чем система Лоренца. Соображения, из которых мы будем выводить соответствующее утверждение, отличаются от соображений Л. П. Шильникова. 
В частности, результат верен и для потоков, имеющих инвариантное локально максимальное гиперболическое множество.

В основе доказательства лежит вариант определения гиперболичности системы, который в работе назван сингулярной гиперболичностью. Система, удовлетворяющая соответствуюшему условию, названа сингулярно гиперболической.

Оказывается, что в случае инвариантного локально максимального множества, не содержащего неподвижных точек, сингулярная гиперболичность эквивалентна классической гиперболичности (инвариантные множества, не содержащие неподвижных точек, могут существовать, если область притяжения не является односвязной; например, является полноторием). Для систем типа системы Лоренца сингулярная гиперболичность эквивалентна гиперболичности отображения последования. Определение сингулярной гиперболичности переносится на неавтономные системы. Свойство сингулярной гиперболичности является грубым относительно малых неавтономных возмущений. Наконец, сингулярно гиперболические системы не могут иметь устойчивых траекторий. Отсюда и из работы [14], в которой доказана гиперболичность отображения последования для системы Лоренца, следует отсутствие устойчивых решений у малых неавтономных возмущений системы Лоренца.

Общая схема работы следуюшая. В $\S 2$ мы повторяем известные факты для уточнения, какие системы рассматриваются как обобщение системы Лоренца, что понимается под аттрактором, что понимается под гиперболичностью и т.д. Попутно доказываются некоторые простые утверждения. В $\S 3$ приводится определение сингулярно гиперболичного потока. Доказываются некоторые свойства сингулярно гиперболических систем. В $\S 4$ доказывается, что для систем типа системы Лоренца гиперболичность отображения последования эквивалентна сингулярной гиперболичности потока. В $\S 5$ формулируется условие сингулярной гиперболичности для неавтономных систем и доказывается его грубость. Наконец, показывается, что из сингулярной гиперболичности следует отсутствие устойчивых траекторий. В $\S 6$ формулируются некоторые дальнейшие свойства сингулярно гиперболических систем, некоторые из них приводятся без доказательства. В частности, сформулированы варианты обобщения на случай произвольной размерности и на случай локально максимального инвариантного множества.

Для сохранения ясности мы ограничимся трехмерным случаем. В случае произвольной размерности будут приведены формулировки результатов.

\section{§2. Системы типа системы Лоренца}

В этом параграфе мы опишем класс рассматриваемых систем, включающий в себя систему Лоренца. Для таких систем приводятся факты, которые в основном хорошо известны.

2.1. Основные обозначения. Через $x$ мы будем обозначать точку $x=\left(x_{1}, x_{2}, x_{3}\right) \in \mathbb{R}^{3}$, через $X(x)$ - векторное поле с координатами $X_{1}(x)$, $X_{2}(x), X_{3}(x)$, определенное в некоторой ограниченной области $U_{0} \subset \mathbb{R}^{3}$. Вектор $X(x)$ мы будем называть вектором скорости. Через $\Phi_{t}$ обозначается локальный поток, порожденньй системой уравнений $\dot{x}=X(x)$. Предполагается, что $U_{0}$ инвариантна относительно $\Phi_{t}$, т.е. $\Phi_{t}\left(U_{0}\right) \subset U_{0}$, если $t>0$. Таким образом, положительная полутраектория любой точки $x \in U_{0}$ целиком лежит в $U_{0}$ и $\Phi_{t}-$ 
полупоток на $U_{0}$.

Через $D(x)$ обозначается матрица из частных производных

$$
D(x)=\left\{\frac{\partial X_{i}}{\partial x_{j}}\right\},
$$

через $d \Phi_{t}$ - дифференциал отображения $\Phi_{t}$. Если мы хотим подчеркнуть, что дифференциал рассматривается только в точке $x$, то применяем обозначение $d \Phi_{t}(x)$. Если $f(x)$ - непрерывная функция, дифференцируемая вдоль траекторий, определенная на $U_{0}$ и принимающая значения в каком-то множестве, то через $\dot{f}$ или $\frac{d}{d t} f(x)$ обозначается производная по направлению векторного поля $X(x)$.

Через $T_{x} U_{0}$ обозначается касательное пространство к $U_{0}$ в точке $x$. Пространство, сопряженное касательному, обозначаем через $T_{x}^{*} U_{0}$. Мы будем рассматривать элементы касательного пространства как вектор-столбцы; элементы пространства, сопряженного касательному пространству, - как вектор-строки. Для векторов $q \in T_{x}^{*} U_{0}, v \in T_{x} U_{0}$ определено скалярное произведение $(q, v)$. Векторы $q \in T_{x}^{*} U_{0}, v \in T_{x} U_{0}$ ортогональны, если $(q, v)=0$.

Некоторая путаница вызвана тем, что в пространстве $\mathbb{R}^{3}$ имеется отождествление касательных пространств во всех точках; кроме того, имеется отождествление касательного пространства и сопряженного к нему. В настоящей работе различаются касательные пространства в разных точках, а также касательное пространство и сопряженное к нему.

В пространствах $T_{x} U_{0}, T_{x}^{*} U_{0}$ определена обычная евклидова норма и связанные с ней скалярное произведение и измерение углов. Длину вектора мы будем обозначать через $|v|$ или $|q|$.

Функцию $q(x)$ со значениями в пространстве $T_{x}^{*} U_{0}$ мы будем называть векторныцм полем (учитывается, что элементы пространства $T_{x}^{*} U_{0}$ - это вектор-строки).

Мы будем рассматривать левые и правые собственные векторы произвольной матрицы $A$. Правый собственный вектор является вектор-столбцом и определяется уравнением $A v=\lambda v$; левый собственный вектор является вектор-строкой и определяется уравнением $q A=\lambda q$. Хорошо известно, что если $q, v-$ левьй и правый собственные векторы, соответствующие различным собственным числам $\lambda_{1}, \lambda_{2}$, то $(q, v)=0$. Соответствуюшее утверждение известно как соотношение ортогональности. Левые собственные векторы рассматриваются как элементы пространства $T_{x}^{*} U_{0}$.

2.2. Неподвижные точки. Мы будем говорить, что неподвижная точка $x_{0}$ имеет mun $(L)$, если собственные числа дифференциала в точке $x_{0}$ (то же самое, что матрицы $\left.D\left(x_{0}\right)\right)$ вешественные и таковы, что $\lambda_{1}<\lambda_{2}<0<\lambda_{3}, \lambda_{2}+\lambda_{3}>0$. Величина $\lambda_{2}+\lambda_{3}$ называется седловой величиной.

Неподвижная точка $x_{0}$ типа $(L)$ имеет двумерное устойчивое многообразие (мы будем обозначать его через $\left.W^{s}\left(x_{0}\right)\right)$ и одномерное неустойчивое многообразие (мы будем обозначать его через $\left.W^{u}\left(x_{0}\right)\right)$. Неустойчивое многообразие разделяется на две сепаратрисы. В устойчивом многообразии выделяется одномерное подмногообразие (мы будем назьвать его сильно устойчивы.м) $W^{s s}\left(x_{0}\right)$; это подмногообразие касается собственного вектора матрицы $D\left(x_{0}\right)$, соответствуюшего собственному значению $\lambda_{1}$, и характеризуется тем, что если точка $x$ принадлежит $W^{s s}\left(x_{0}\right)$, то $\left|x_{0}-\Phi_{t}(x)\right|<c e^{\lambda_{1} t}$ для некоторой константы $c>0$ и любого $t<0$. 
Мы будем говорить, что неподвижная точка $x_{0}$ имеет mun $(F)$, если матрища $D\left(x_{0}\right)$ (определенная в (3)) имеет одно вешественное собственное значение $\lambda_{1}<0$ и пару чисел $\lambda_{2}, \lambda_{3}$, причем $\operatorname{Re} \lambda_{2}>0, \operatorname{Re} \lambda_{3}>0$. Числа $\lambda_{2}, \lambda_{3}$ могут быть вешественными или парой комплексно-сопряженных. Неподвижная точка $x_{0}$ типа $(F)$ имеет двумерное неустойчивое многообразие (мы будем обозначать его через $\left.W^{u}\left(x_{0}\right)\right)$ и одномерное устойчивое многообразие (мы будем обозначать его через $\left.W^{s}\left(x_{0}\right)\right)$. Устойчивое многообразие разделяется на две сепаратрисы.

Рассмотрение именно этих двух типов неподвижных точек обосновывается тем, что, во-первых, в системе Лоренца при указанных значениях параметров имеются неподвижные точки именно этих двух типов; во-вторых, в определенных ниже сингулярно гиперболических системах размерности 3 имеются только такие неподвижные точки, если нет собственных чисел с нулевой вешественной частью.

Система Лоренца имеет неподвижную точку $O(0,0,0)$ типа $(L)$ и две неподвижные точки $A_{ \pm}( \pm \sqrt{b(r-1)}, \pm \sqrt{b(r-1)}, r-1)$ типа $(F)$. Матрица $D\left(A_{ \pm}\right)$имеет одно вешественное собственное число $\lambda_{1}<0$ и пару комплексно-сопряженных $\lambda_{2}, \bar{\lambda}_{2}, \operatorname{Re} \lambda_{2}>0$.

Известно, что поток в окрестности неподвижной точки $x_{0}$ приводится гладким преобразованием к линейному, если нет резонансов низкого порядка [18], [19]. Если в окрестности неподвижной точки систему координат $\left(y_{1}, y_{2}, y_{3}\right)$ выбрать в соответствии с базисом из собственных векторов, то $W^{s s}\left(x_{0}\right)$ совпадает с осью $O Y_{1}$.

Нам потребуется более слабое утверждение, для которого не надо требовать отсутствия резонансов [20].

Лемма 2.1. В окрестности неподвижной точки $x_{0}$ типа $(L)$ существует такая система координат $\left(y_{1}, y_{2}, y_{3}\right)$, в которой неподвижная точка имеет координаты $(0,0,0)$, устойчивое многообразие $W^{s}\left(x_{0}\right)$ определяется уравнением $y_{1}=0$, неустойчивое - уравнениями $y_{2}=0, y_{3}=0$. Система уравнений в этой системе координат имеет вид

$$
\dot{y}_{i}=\lambda_{i} y_{i}+f_{i}(y), \quad i=1,2,3,
$$

где разложсение в ряд Тейлора функиий $f_{i}(y)$ начинается с членов второго порядка малости.

ДокАЗАТЕЛЬСтво. Пусть $x_{0}$ - неподвижная точка типа $(L)$. Перенесем начало координат в точку $x_{0}$ и сделаем линейную замену координат $x=B u$ с матрицей $B$ так, что матрица $D\left(x_{0}\right)$ приводится к диагональному виду. В этой системе уравнение имеет вид $\dot{u}_{i}=\lambda_{i} u_{i}+g_{i}(u)$, где разложение в ряд Тейлора функций $g_{i}(u)$ начинается с членов второго порядка по переменной $u$. Устойчивое многообразие $W^{s}\left(x_{0}\right)$ определяется уравнением $u_{3}=\psi\left(u_{1}, u_{2}\right)$, причем разложение в ряд Тейлора функции $\psi\left(u_{1}, u_{2}\right)$ начинается с членов второго порядка (это следует из того, что $W^{s}\left(x_{0}\right)$ касается плоскости $\left.u_{3}=0\right)$. Положим $s_{1}=u_{1}, s_{2}=u_{2}$, $s_{3}=u_{3}-\psi\left(u_{1}, u_{2}\right)$. В системе координат $\left(s_{1}, s_{2}, s_{3}\right)$ неустойчивое многообразие имеет вид $s_{1}=\varphi_{1}\left(s_{3}\right), s_{2}=\varphi_{2}\left(s_{3}\right)$. Положим $y_{1}=s_{1}-\varphi_{1}\left(s_{3}\right), y_{2}=s_{2}-\varphi_{2}\left(s_{3}\right)$, $y_{3}=s_{3}$. Это и есть нужная система координат. Лемма доказана.

Аналогично можно показать, что в окрестности неподвижной точки $x_{0}$ типа $(F)$ существует такая система координат $\left(y_{1}, y_{2}, y_{3}\right)$, что система в случае вешественных собственных чисел матрицы $D\left(x_{0}\right)$ имеет вид

$$
\dot{y}_{i}=\lambda_{i} y_{i}+f_{i}(y), \quad i=1,2,3, \quad y=\left(y_{1}, y_{2}, y_{3}\right),
$$


а в случае вещественного числа $\lambda_{1}$ и пары комплексно-сопряженных собственных чисел $\lambda_{2}, \bar{\lambda}_{2}$ имеет вид

$$
\dot{y}_{1}=\lambda_{1} y_{1}+f_{1}\left(y_{1}, z\right), \quad \dot{z}=\lambda_{2} z+f_{2}\left(y_{1}, z\right)
$$

где $z$ - комплексная переменная $z=y_{2}+i y_{3}$, разложение функций $f_{1}, f_{2}$ в ряд Тейлора начинается с членов второго порядка, неустойчивое многообразие определяется уравнением $y_{1}=0$, устойчивое многообразие определяется уравнениями $y_{2}=0, y_{3}=0$.

2.3. Определение системы типа системы Лоренца. Пусть $P$ - поверхность, краем которой является объединение конечного числа замкнутых контуров (не обязательно связная), не содержащая неподвижных точек и такая, что вектор скорости $X(x)$ во всех точках поверхности $P$ (включая край поверхности $P$ ) трансверсален $P$ (напомним, что трансверсальность в данном случае означает, что угол между $X(x)$ и поверхностью $P$ ненулевой).

Касательное пространство к поверхности $P$ в точке $x$ обозначается через $T_{x} P$. Отображение последования на поверхности $P$ обозначается через $F_{P}$ (или через $F$, если ясно, о какой поверхности $P$ идет речь).

Отображение $F_{P}$, как правило, определено не на всей поверхности $P$, а только на ее части, так как на $P$ имеются точки, положительные полутраектории которых не пересекаются с $P$. Это, в частности, точки, принадлежащие пересечению устойчивого многообразия неподвижной точки и поверхности $P$.

ОПрЕДЕЛЕНИЕ 2.2. Динамическая система, порожденная векторнњм полем $X(x)$ в некоторой инвариантной области $U_{0} \subset \mathbb{R}^{3}$, называется системой типа системы Лоренца, если вьполнено следующее.

УсловиЕ (L 1$)$. В области $U_{0}$ имеется не более конечного числа неподвижных точек $x_{1}, \ldots, x_{m}$; все неподвижные точки являются точками типа $(L)$ или $(F)$.

Условие (L2). Существует поверхность $P$ (не обязательно связная), край которой состоит из конечного числа простых замкнутых контуров, не содержащая неподвижных точек и такая, что вектор скорости в каждой точке $x \in P$ трансверсален $P$. При этом угол между вектором скорости и плоскостью, касательной к $P$, равномерно отделен от нуля.

УСловие (L3). Многообразия $W^{s s}\left(x_{j}\right)$ неподвижных точек типа $(L)$ и многообразия $W^{s}\left(x_{j}\right)$ неподвижных точек типа $(F)$ не пересекаются с поверхностью $P$ и не лежат целиком в $U_{0}$.

УСловиЕ (L4). Всякая положительная полутраектория из области $U_{0}$, кроме неподвижных точек и полутраекторий, лежащих на устойчивых многообразиях неподвижных точек, пересекается с $P$. Всякая положительная полутраектория точки $x \in P$, включая край поверхности $P$, возвращается обязательно во внутреннюю точку поверхности $P$. Неустойчивые многообразия неподвижных точек типа $(L)$ пересекаются с внутренностью поверхности $P$.

Для конкретной системы Лоренца поверхность $P$-это часть плоскости $z=r-1$, ограниченная кривьми $x y=b(r-1)-\varepsilon$ с некоторьм достаточно мальм числом 
$\varepsilon>0$ и кривыми $|x-y|=50$. На кривой $x y=b(r-1)$ вектор скорости касается плоскости $z=r-1$.

Приведем некоторые свойства отображения $F_{P}$.

1) Если $x \in P$ и положительная полутраектория точки $x$ пересекается с $P$, то $F_{P}$ является гладким в окрестности точки $x$ (и даже аналитическим, если поверхность и векторное поле $X(x)$ являются аналитическими). Это следует из теорем о гладкой и аналитической зависимости решений от начальных условий и из того, что полутраектория точки $x$ попадает во внутреннюю точку поверхности $P$.

2) Из условия (L3) следует, что если положительная полутраектория точки $x \in P$ не пересекается с поверхностью $P$, то $x$ принадлежит устойчивому многообразию некоторой неподвижной точки $x_{j}$ типа $(L)$. При этом точка $x$ не принадлежит многообразию $W^{s s}\left(x_{j}\right)$ неподвижной точки $x_{j}$ типа $(L)$ и не принадлежит устойчивому многообразию неподвижной точки типа $(F)$. Так как $X(x)$ трансверсально $P$ и касается многообразия $W^{s}\left(x_{j}\right)$, то многообразия $P$ и $W^{s}\left(x_{j}\right)$ трансверсальны. Следовательно, $W^{s}\left(x_{j}\right) \cap P$ является гладким многообразием размерности 1. Поэтому пересечение поверхности $P$ со всеми устойчивьми многообразиями всех неподвижных точек типа $(L)$ (обозначим это множество через $\Gamma$ ) является объединением некоторого количества гладких кривых. Эти кривые не пересекаются, так как устойчивые многообразия неподвижных точек не могут пересекаться.

3) В точках множества Г отображение $F_{P}$ не определено и разрывно. Отображение $F_{P}^{-1}$ также определено только на некотором подмножестве (именно на $\left.F_{P}(P \backslash \Gamma)\right)$. Аналогично, $F_{P}^{n}, F_{P}^{-n}$ определены только на некоторых подмножествах (не будем уточнять, на каких).

Мы будем употреблять обозначения $F_{P}^{n}(A), F_{P}^{-n}(A)$, подразумевая под этими обозначениями $F_{P}^{n}(A \cap M), F_{P}^{-n}(A \cap N)$, где $M, N$ - множества, на которых определены соответственно $F_{P}^{n}$ или $F_{P}^{-n}$.

2.4. Аттрактор. В литературе имеются различные определения термина "аттрактор". Обычно под аттрактором потока $\Phi_{t}$ понимают замкнутое инвариантное множество $\Lambda$, к которому притягиваются положительные полутраектории всех точек из некоторой окрестности множества $\Lambda$. Следовательно, для заданного потока в некоторой окрестности $U_{0}$ может быть несколько аттракторов. Поэтому вопрос о том, что понимается под аттрактором конкретного потока, определяемого системой Лоренца, требует уточнения. Мы будем рассматривать два аттрактора. Один из них назовем глобальным аттрактором. Другой аттрактор - это тот аттрактор, который мы понимаем под аттрактором Лоренца. Отметим, что и рассматриваемый ниже аттрактор системы тоже может быть не окончательньм, он может содержать еше один или несколько более малых аттракторов.

Глобальнымм аттрактором мы будем называть множество $\Lambda_{g}=\bigcap_{t>0} \Phi_{t}\left(U_{0}\right)$. Очевидно, что $\Lambda_{g}$ является инвариантным множеством и каждая положительная полутраектория $x(t), t>0$, притягивается к $\Lambda$, если $x(0) \in U_{0}$. Точки множества $\Lambda_{g}$ характеризуются тем, что полная траектория $x(t), t \in \mathbb{R}^{1}$, принадлежит $U_{0}$, если $x(0) \in \Lambda_{g}$. Отсюда следует, что вся траектория $x(t)$ принадлежит $\Lambda_{g}$, если $x(0) \in \Lambda_{g}$. В частности, неподвижные точки, принадлежащие $U_{0}$, и их неустойчивые многообразия принадлежат $\Lambda_{g}$. 
Аттрактором отображсения $F_{P}$ назовем множество

$$
\Lambda_{P}=\operatorname{clos}\left(\bigcap_{n=1}^{\infty} F_{P}^{n}(P)\right)
$$

(через $\operatorname{clos} A$ обозначается замыкание множества $A$ ).

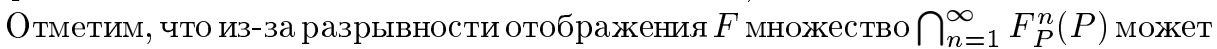
быть незамкнутым. Поэтому в определении мы берем замькание множества.

Aттрактором (или аттрактором потока, если необходимо подчеркнуть отличие от $\Lambda_{P}$ ) будем называть замыкание множества всех положительных полутраекторий точек $x \in \Lambda_{P}$. Обозначать аттрактор мы будем через $\Lambda$. Можно показать, что $\Lambda$ состоит из траекторий точек $x \in \Lambda_{P}$, неподвижных точек типа $(L)$ и их неустойчивых многообразий. Кроме того, $\Lambda_{P}=\Lambda \cap P$. Это будет видно из доказательства следующей леммы, которая утверждает, что $\Lambda$ - аттрактор потока в смысле приведенного выше определения.

Лемма 2.3. Существует такая окрестность $U$ множсества $\Lambda$, что

$$
\Phi_{t}(U) \subset U, \quad t>0, \quad \Lambda=\bigcap_{t>0} \Phi_{t}(U) .
$$

ДокаЗАтельство. Рассмотрим множество $U_{1}$, состоящее из положительных полутраекторий всех точек $x \in P$. Покажем, что граница $\partial U_{1}$ множества $U_{1}$ состоит из точек $x \in P \backslash F_{P}(P)$, положительных полутраекторий точек $x \in \partial P$ до их пересечений с поверхностью $P$, неподвижных точек типа $(L)$ и их неустойчивых многообразий.

Действительно, пусть последовательность точек $x_{n} \in U_{1}$ сходится к точке $x_{\infty}$. Точки $x_{n}$ лежат на положительных полутраекториях некоторых точек $x_{n}^{\prime} \in P$. Пусть соответствуюшие полутраектории $x_{n}(t)$ выбраны так, что $x_{n}(0)=x_{n}^{\prime}$, $x_{n}\left(t_{n}\right)=x_{n}, x_{n}(t) \notin P$, если $t \in\left(0, t_{n}\right)$. В силу компактности $P$ из последовательности $x_{n}^{\prime}$ можно выделить сходящуюся подпоследовательность. Можно предположить, что сама последовательность $x_{n}^{\prime}$ сходится к некоторой точке $x_{\infty}^{\prime}$. Возможны следующие случаи.

1) Последовательность $t_{n}$ ограничена, $x_{\infty}^{\prime} \in \partial P$. В этом случае точка $x_{\infty}$ лежит на части положительной полутраектории точки $x_{\infty}^{\prime} \in \partial P$ до пересечения с $P$ и, очевидно, принадлежит границе $U_{1}$.

$2)$ Последовательность $t_{n}$ ограничена, $x_{\infty}^{\prime} \notin \partial P$. В этом случае $x_{\infty}-$ внутренняя точка множества $U_{1}$.

3) Последовательность $t_{n}$ не ограничена. В этом случае по соображениям непрерывности $t_{n} \rightarrow \infty$ и потому полутраектория точки $x_{\infty}^{\prime}$ не пересекается с поверхностью $P$; из условия (L4) следует, что $x_{\infty}^{\prime}$ принадлежит устойчивому многообразию некоторой неподвижной точки $x_{j}$ типа $(L)$, а точка $x_{\infty}$ принадлежит пролонгации положительной полутраектории точки $x_{\infty}^{\prime}$. Как известно, пролонгация состоит из неподвижной точки $x_{j}$ и ее неустойчивого многообразия $W^{u}\left(x_{j}\right)$. Точка $x_{j}$ и $W^{u}\left(x_{j}\right)$ не принадлежат $U_{1}$; следовательно, они принадлежат границе $U_{1}$.

Таким образом, мы показали, что предельньми точками множества $U_{1}$ являются точки множества $U_{1}$, точки, лежащие на положительных полутраекториях точек $x \in \partial P$, неподвижные точки типа $(L)$ и их неустойчивые многообразия. 
Граничными могут быть неподвижные точки типа $(L)$, отрезки их неустойчивых многообразий до пересечения с $P$, отрезки положительных полутраекторий точек $x \in \partial P$ до пересечения с $P$ и точки $x \in P$. Точки множества $F_{P}(P)$ являются внутренними для $U_{1}$.

Отметим, что неподвижные точки и их неустойчивые многообразия не могут принадлежать внутренности $U_{1}$, так как имеется траектория (например, $W^{s s}\left(x_{j}\right)$ ), не пересекающаяся с $P$ и подходящая как угодно близко к $x_{j}$.

Построим специальную окрестность неподвижной точки $x_{j}$ типа $(L)$. Для этого перейдем к системе координат $\left(y_{1}, y_{2}, y_{3}\right)$ в окрестности точки $x_{j}$, определяемой леммой 2.1. Зададим множество $V_{j, \delta, r}$ неравенствами $y_{1}^{2}+y_{2}^{2} \leqslant r,\left|y_{3}\right| \leqslant \delta$. Назовем кольцом множество, определенное уравнением $y_{1}^{2}+y_{2}^{2}=r,\left|y_{3}\right| \leqslant \delta$, кругом множество $\left\{y_{1}^{2}+y_{2}^{2} \leqslant r, y_{3}=\delta\right\}$ или $\left\{y_{1}^{2}+y_{2}^{2} \leqslant r, y_{3}=-\delta\right\}$. Очевидно, что граница множества $V_{j, \delta, r}$ состоит из кольца и двух кругов, и если $\delta, r$ достаточно малы, то через кольцо траектории входят в $V_{j, \delta, r}$, через круги выходят из $V_{j, \delta, r}$.

Определим множество $U$ как объединение множества $U_{1}$, множеств $V_{j, \delta, r}$ и отрезков положительных полутраекторий точек множества $V_{j, \delta, r}$ до пересечения с $P$. Очевидно, что если $r$ и $\delta$ достаточно малы, то положительные полутраектории точек из $V_{j, \delta, r}$ пересекаются с поверхностью $P$ и не пересекаются с краем поверхности $P$ (последнее утверждение следует из того, что неустойчивые многообразия неподвижных точек типа $(L)$ пересекаются с внутренностью поверхности $P$ и не пересекаются с краем $P$ ). Нужные свойства множества $U$ очевидны.

Лемма доказана.

2.5. Гиперболичность. Под гиперболичностью системы типа системы Лоренца обычно понимают гиперболичность отображения последования на поверхности $P$. Сформулируем два варианта соответствуюшего определения.

Как было сказано выше, отображение последования на поверхности $P$ является разрывным (разрывы имеются на множестве $\Gamma$, которое является объединением гладких непересекающихся линий). В остальных точках отображение $F_{P}$ является гладким (и даже аналитическим, если поверхность $P$ аналитична и система уравнений задана аналитическими функциями). Поэтому мы приведем модификацию общего определения гиперболичности применительно к рассматриваемому случаю.

Именно, пусть $F$ - отображение двумерного многообразия $P$ с краем $\partial P$, имеюшее разрывы на множестве $Г$. Множество Г является объединением конечного числа гладких непересекающихся кривых.

ОПРЕДЕЛЕНИЕ 2.4. Конусом в евклидовом пространстве с осевым пространством $Q$ и раствором $\alpha$ называется множество таких векторов $v$, что $\angle(v, Q)<\alpha$ (через $\angle(v, Q)$ обозначается угол между вектором $v$ и пространством $Q)$.

ОпРеДЕЛЕниЕ 2.5. Отображение $F$ называется гиперболичным, если выполнено следующее.

УСловие (HU). Для каждой точки $x \in P$ определены конусы $\mathscr{K}_{x}^{u} \subset T_{x} P$, $\mathscr{K}_{x}^{s} \subset T_{x} P$, непрерывно зависяшие от точки $x$. Для конусов $\mathscr{K}_{x}^{u}, \mathscr{K}_{x}^{s}$ выполнены условия:

a) если $x \in P \backslash \Gamma$, то $\cos \left(d F\left(\mathscr{K}_{x}^{u}\right)\right) \subset \operatorname{int}\left(\mathscr{K}_{F(x)}^{u}\right)$ (здесь и далее через $\operatorname{clos}$ и 
int обозначены замыкание и внутренность множества); если $x \in F(P \backslash \Gamma)$, то $\operatorname{clos}\left(d F^{-1}\left(\mathscr{K}_{x}^{s}\right)\right) \subset \operatorname{int}\left(\mathscr{K}_{F^{-1}(x)}^{s}\right) ;$

b) существуют такие константы $c>0, \mu>1$, что

$\mathrm{bu)}$ для любой точки $x \in P$, для любого вектора $v \in \mathscr{K}_{x}^{u}$ и любого натурального $n$, для которого $F^{n}(x)$ определено, выполнено неравенство $\left|d F^{n}(v)\right|>c \mu^{n}|v|$

bs) для любой точки $x \in P$, любого вектора $v \in \mathscr{K}_{x}^{s}$ и любого натурального $n$, для которого $F^{-n}(x)$ определено, выполнено неравенство $\left|d F^{-n}(v)\right|>c \mu^{n}|v|$

c) касательная к множеству $\Gamma$ в каждой точке $x \in \Gamma$ лежит в конусе $\mathscr{K}_{x}^{s}$.

Конус $\mathscr{K}_{x}^{u}$ называется неустойчивым конусом, конус $\mathscr{K}_{x}^{s}-$ устойчивым конусом.

Второй вариант определения гиперболичности.

УС ловиЕ $(\mathrm{H} \Lambda)$. Для каждой точки $x \in \Lambda_{P}$ определено разложение пространства $T_{x} P$ в прямую сумму $T_{x} P=E_{x}^{u} \oplus E_{x}^{s}$. Пространства $E_{x}^{u}, E_{x}^{s}$ инвариантны, непрерывно зависят от $x$ на $\Lambda_{P}$ и удовлетворяют следующим условиям:

а) сушествует такая константа $\delta>0$, что если $v_{1} \in E_{x}^{u}, v_{2} \in E_{x}^{s}$, то справедливо неравенство $\angle\left(v_{1}, v_{2}\right)>\delta$;

b) сушествуют такие константы $c>0, \mu>0$, что

bu) если $x \in \Lambda_{P}, v \in E_{x}^{u}, F^{n}(x)$ определено, то $\left|d F^{n}(v)\right|>c \mu^{n}|v|$;

$\mathrm{bs}$ ) если $x \in \Lambda_{P}, v \in E_{x}^{s}, F^{-n}(x)$ определено, то $\left|d F^{-n}(v)\right|>c \mu^{n}|v|$;

c) если $x \in \Gamma$, то $E_{x}^{s}$ касается $Г$.

Здесь константы $c, \mu$ те же самые, что и в условии (HU).

Известно, что условия (HU), (НА) для гладких отображений (без разрывов) с локально максимальньм множеством $\Lambda_{P}$ эквивалентны. Именно, если для гладкого отображения (без разрывов) в окрестности инвариантного локально максимального множества $\Lambda_{P}$ выполнено условие (HU), то на множестве $\Lambda_{P}$ выполнено условие $(\mathrm{H} \Lambda)$. Опишем процесс построения пространств $E_{x}^{u}, E_{x}^{s}$ для общего отображения $F$ (не обязательно отображения последования на поверхности $P$ ).

Предположим, что вьполнено условие (HU). Определим пространства $E_{x}^{s}$ для тех точек $x \in P$, для которых $F^{n}(x)$ определено при всех натуральных $n$. Это те точки, для которых $x \notin \Gamma, F^{n}(x) \notin \Gamma$ при всех натуральных $n$. Положим

$$
E_{x}^{s}=\bigcap_{n=1}^{\infty} d F^{-n}\left(\mathscr{K}_{F^{n}(x)}^{s}\right) .
$$

Семейство пространств $E_{x}^{s}$ является инвариантным, непрерывно зависит от точки $x$ и продолжается на весь аттрактор $\Lambda_{P}$ по непрерывности. Для систем типа системы Лоренща в точках $x \in \Gamma$ пространство $E_{x}^{s}$ совпадает с касательной к кривой Г. Более того, на $P$ сушествует семейство устойчивых многообразий, т.е. кривых, которые в каждой точке касаются пространства $E_{x}^{s}$. Доказательство этих утверждений можно найти, например, в [7] (в этой статье условия формально отличаются от приведенных в настоящей статье, но по существу близки к вышеприведенным; все доказательства без труда переносятся на рассматриваемый в настоящей работе случай). 
Аналогично, для каждой точки $x \in \Lambda$, для которой $F^{-n}(x)$ определено при всех натуральных $n$, определено пространство $E_{x}^{u}$, назьваемое неустойчивым пространством:

$$
E_{x}^{u}=\bigcap_{n=1}^{\infty} d F^{n}\left(\mathscr{K}_{F^{-n}(x)}^{u}\right) .
$$

Это пространство определено на множестве $\bigcap_{n=1}^{\infty} F^{n}(P)$ (т.е. на части аттрактора $\Lambda$ ) и продолжается по непрерьвности на весь аттрактор $\Lambda$. В точке $x \in \Gamma$ пространство $E_{x}^{u}$ трансверсально $\Gamma$.

Наоборот, если выполнено условие (Н $\Lambda$ ), то существует окрестность множества $\Lambda_{P}$, на которой выполнено условие (HU). В качестве конусов $\mathscr{K}_{x}^{u}, \mathscr{K}_{x}^{s}$ можно выбрать достаточно малые окрестности пространств $E_{x}^{u}, E_{x}^{s}$, которые продолжаются с сохранением непрерьвности на окрестность множества $\Lambda_{P}$.

ЗАмечАниЕ. Вообше говоря, условия (HU) и (НА) для общих систем, имеющих разрывы, скорее всего, неэквивалентны. Для отображения последования на поверхности $P$ системы типа системы Лоренца условия (HU) и (Н $\Lambda)$ эквивалентны, однако это не такой тривиальный факт, как для гладких отображений без особенностей.

Приведем классическое определение гиперболичности потока на инвариантном множестве. Это определение формулируется для аттрактора, но оно переносится дословно на любое инвариантное замкнутое множество.

Пусть поток задан уравнением $\dot{x}=X(x)$ на некотором открытом множестве $U$, содержащем аттрактор $\Lambda$.

ОПРЕДЕЛЕНИЕ 2.6. Поток $\Phi_{t}$ әиперболичен на инвариантном множсестве $\Lambda \subset U$, не содержашем неподвижных точек, если для каждой точки $x \in \Lambda$ имеется разложение пространства $T_{x} U$ в прямую сумму $T_{x} U=E_{x}^{u} \oplus E_{x}^{0} \oplus E_{x}^{s}$. Пространства $E_{x}^{u}, E_{x}^{s}, E_{x}^{0}$ инвариантны относительно $d \Phi_{t}$, непрерывно зависят от точки $x \in \Lambda$ и дифференцируемы вдоль траекторий. Пространство $E_{x}^{0}$ порождено вектором $X(x)$. Кроме того, сушествуют такие константы $c>0, \gamma>0$, что

a) если $v \in E_{x}^{u}$, то $\left|d \Phi_{t}(v)\right|>c e^{\gamma t}|v|, t>0$;

b) если $v \in E_{x}^{s}$, то $\left|d \Phi_{-t}(v)\right|>c e^{\gamma t}|v|, t>0$.

Если у системы типа системы Лоренца имеются неподвижные точки типа $(L)$, то поток не гиперболичен в смысле последнего определения.

В заключение докажем еще одно известное утверждение.

У реальной системы Лоренца $\operatorname{div} X(x)<0$, поэтому наличие устойчивого конуса следует из наличия неустойчивого. Это видно из следуюшего утверждения, которое в литературе неоднократно встречалось, но ввиду простоты иногда даже не формулировалось.

ЛЕмма 2.7. Пусть для системъ типа системы Лоренца выполнены следующие условия: $\operatorname{div} X<0$ и для отображения $F_{P}$ существует семейство неустойчивых конусов $\mathscr{K}_{x}^{u}$, для которых выполнены условия инвариантности и bu) из условия (HU). Тогда существует семейство устойчивых конусов $\mathscr{K}_{x}^{s}$, для которых выполнены условия инвариантности и $\mathrm{bs}$ ) из условия (HU). 
ДокаЗАТЕЛЬСтво. В качестве семейства конусов $\mathscr{K}_{x}^{s} \subset T_{x} P$ рассмотрим конусы, дополнительные к конусам $\mathscr{K}_{x}^{u}$. Так как конус $\mathscr{K}_{x}^{u}$ сужается под действием $F$, то конусы $\mathscr{K}_{x}^{s}$ сужаются под действием $F^{-1}$. Из существования неустойчивых конусов следует сушествование семейства пространств $E_{x}^{u}, x \in \Lambda \cap P$. Пусть $x \in \Lambda \cap P, v_{1} \in E_{x}^{u}, v_{2} \in \mathscr{K}_{x}^{s}$. Из условия $\operatorname{div} X<0$ следует, что функция $V_{n}\left(x, v_{1}, v_{2}\right)$, равная объему параллелепипеда, порожденного векторами $d F_{P}^{n}\left(v_{1}\right)$, $d F_{P}^{n}\left(v_{2}\right), X\left(F_{P}^{n}(x)\right)$, экспоненциально убьвает с ростом $n$. Так как $X(x)$ ограничено на $P$, то площадь параллелограмма, порожденного векторами $d F_{P}^{n}\left(v_{1}\right)$, $d F_{P}^{n}\left(v_{2}\right)$, экспоненциально убывает с ростом $n$. Следовательно, плошадь параллелограмма, порожденного векторами $d F_{P}^{-n}\left(v_{1}\right), d F_{P}^{-n}\left(v_{2}\right)$ (если $d F_{P}^{-n}$ определено), экспоненциально растет. Но если $v_{1} \in E_{x}^{u}$, то $d F_{P}^{-n}\left(v_{1}\right)$ экспоненциально убывает. Следовательно, $d F_{P}^{-n}\left(v_{2}\right)$ экспоненциально растет. Отсюда следует оценка $\left|d F_{P}^{-n}\left(v_{2}\right)\right|>c \mu_{2}^{n}$ при некоторых константах $c>0, \mu_{2}>1$. Лемма доказана.

\section{§3. Сингулярно гиперболическая структура}

3.1. Уравнение в вариациях и сопряженное уравнение в вариациях. Далее рассматривается известное уравнение в вариациях. Если $x(t)$ - траектория, то уравнение в вариациях вдоль траектории $x(t)$ имеет вид $\dot{v}=D(x(t)) v$. В этом уравнении вектор $v(t)$ представляется как вектор-столбец. Вместе с уравнением в вариациях мы будем рассматривать сопряженное уравнение в вариациях вдоль траектории $x(t)$, имеюшее вид $\dot{q}=-q D(x(t))$. В этом уравнении вектор $q$ представляется как вектор-строка, т.е. как элемент пространства $T_{x}^{*} U$.

Понимать уравнение в вариациях и сопряженное уравнение в вариациях нужно так. У нас имеется стандартное отождествление пространства $T_{x} U$ с пространством $\mathbb{R}^{3}$. В соответствии с этим отождествлением мы можем написать уравнение с решением $v(t) \in \mathbb{R}^{3}$. Затем для каждого $t$ вектор $v(t)$ отождествляется с вектором из пространства $T_{x(t)} U$.

Хорошо известно, что дифференциал $d \Phi_{t}$ потока $\Phi_{t}$ связан с уравнением в вариациях. Именно, если $v(t)$ - решение уравнения в вариациях вдоль траектории $x(t)$, то $d \Phi_{t} v(0)=v(t)$.

С сопряженным уравнением в вариациях свяжем оператор $d \Phi_{t}^{*}$, который назовем сопряжсенным дифференциалом. Оператор $d \Phi_{t}^{*}$ определяется в пространстве $T_{x}^{*} U$ и принимает значения в пространстве $T_{x(t)}^{*} U$ так, что если $q(t)-$ решение сопряженного уравнения в вариациях вдоль траектории $x(t), x(0)=x$, то $q(0) d \Phi_{t}^{*}=q(t)$.

Действие оператора $d \Phi_{t}^{*}$ записьвается справа по той причине, что такая запись согласуется с обычным определением произведения матриц (произведение вектор-строки на $(3 \times 3)$-матрицу). Кроме того, по существу действие группы операторов $d \Phi_{t}^{*}$ является правым.

Лемма 3.1. Пусть $v(t), q(t)$ - решения уравнения в вариациях и сопряженного уравнения в вариациях вдоль одной и той же траектории $x(t)$. Тогда $(q(t), v(t))=$ const. 
ДоКАЗАТЕЛЬСТво. Справедливость леммы следует из очевидной цепочки равенств

$$
\frac{d}{d t}(q, v)=(\dot{q}, v)+(q, \dot{v})=(-q D(x(t)), v)+(q, D(x(t)) v)=0 .
$$

Лемма доказана.

Подобные утверж дения встречались неоднократно. Имеется подобное утверждение, например, в [16]. Мы формулируем это утверждение в виде отдельной леммы в силу того, что ниже она неоднократно используется.

СлеДСтвИЕ. Для любых $v \in T_{x} U, q \in T_{x}^{*} U$ справедливо равенство

$$
\left(q d \Phi_{t}^{*}, d \Phi_{t} v\right)=(q, v) .
$$

Из последнего соотношения видно, что оператор $d \Phi_{t}^{*}$ является обратным к оператору, сопряженному оператору $d \Phi_{t}$.

3.2. Определение сингулярно гиперболической структуры. Мы приведем определение сингулярно гиперболической системы для более общих систем, чем системы типа системы Лоренца. Именно, пусть в открытом ограниченном множестве $U \subset \mathbb{R}^{3}$ определено гладкое векторное поле $X(x)$, порождающее локальный поток $\Phi_{t}$. Через $\Lambda$ мы будем обозначать множество таких точек, что полная траектория, проходящая через эту точку, целиком принадлежит $U$. Если положительная полутраектория каждой точки $x \in U$ целиком принадлежит $U$, то $\Lambda$ аттрактор.

Предполагается, что $X(x)$ не определено за пределами $U$.

ОПРЕДЕЛЕНИЕ 3.2. На $U$ определена сингулярно гиперболическая структу$p a$, а система называется сингулярно гиперболической, если выполнено

УСловие $\left(\mathrm{H}^{*} \mathrm{U}\right)$. Для каждой точки $x \in U$ определен конус $\mathscr{K}_{x}^{u *} \subset T_{x}^{*} U$ с одномерным осевым пространством $Q_{x}^{*}$; семейство конусов инвариантно в том смысле, что сушествует такое $t_{1}>0$, что если $t>t_{1}$, то $\cos \left(\mathscr{K}_{x}^{u *} d \Phi_{t}^{*}\right) \subset \operatorname{int}\left(\mathscr{K}_{\Phi_{t}(x)}^{u *}\right)$. Кроме того, сушествуют такие константы $c_{1}>0, \gamma_{1}>0, c_{2}>0, \gamma_{2}>0$, что

а) для любой точки $x \in U$, любого вектора $q \in \mathscr{K}_{x}^{u *}$ и любого $t>0$, для которого $\Phi_{t}$ определено, справедливо неравенство $\left|q d \Phi_{t}^{*}\right|>c_{1} e^{\gamma_{1} t}|q|$;

b) для любой точки $x \in U$, любых векторов $v_{1}, v_{2}$, ортогональных некоторому вектору $q \in \mathscr{K}_{x}^{u *}$, и любого $t>0$, для которого $\Phi_{t}$ определено, справедливо неравенство

$$
S\left(d \Phi_{t} v_{1}, d \Phi_{t} v_{2}\right)>c_{2} e^{\gamma_{2} t} S\left(v_{1}, v_{2}\right),
$$

где $S\left(v_{1}, v_{2}\right)$ (соответственно $\left.S\left(d \Phi_{t} v_{1}, d \Phi_{t} v_{2}\right)\right)$ - плошадь параллелограмма, порожденного векторами $v_{1}, v_{2}$ (соответственно $\left.d \Phi_{t} v_{1}, d \Phi_{t} v_{2}\right)$.

Отметим, что если векторы $v_{1}, v_{2}$ ортогональны вектору $q$, то в силу леммы 3.1 векторы $v_{1}(t)=d \Phi_{t} v_{1}, v_{2}(t)=d \Phi_{t} v_{2}$ ортогональны вектору $q(t)=q d \Phi_{t}$ при любом $t$.

Попытаемся объяснить смысл условия $\left(\mathrm{H}^{*} \mathrm{U}\right)$.

Для потока, порож денного системой Лоренца, условия гиперболичности потока не выполняются из-за того, что в аттракторе имеется неподвижная точка. Условие 
гиперболичности формулируется для отображения последования. Поэтому основная идея состоит в замене пространств $E_{x}^{0}, E_{x}^{u}$ на одно пространство $E_{x}=E_{x}^{0} \oplus E_{x}^{u}$, а вместо роста вектора в одном из двух направлений рассмотреть что-то другое. Этим другим является рост площади параллелограмма, лежащего в этом пространстве. В случае сушествования инвариантного разложения $E_{x}=E_{x}^{0} \oplus E_{x}^{u}$ из-за ограниченности вектора $X(x)$ условие роста площади эквивалентно условию роста вектора в направлении $E_{x}^{u}$. Но в трехмерном пространстве плоскость определяется ее нормалью. Нормаль следует представлять как вектор из сопряженного пространства. Поэтому условие существования инвариантного семейства плоскостей мы формулируем в виде условия сушествования инвариантного семейства нормалей к этим плоскостям, что вызвано соображениями удобства. Для того чтобы условие было грубым, мы формулируем его в виде условия сохранения конусов.

Если в области $U$ выполнено условие $\left(\mathrm{H}^{*} \mathrm{U}\right)$, то выполнено следующее

Условие $\left(\mathrm{H}^{*} \Lambda\right)$. На множестве $\Lambda$ имеется инвариантное семейство подпространств $E_{x}^{u *} \subset T_{x}^{*}$. Пространства $E_{x}^{u *}$ одномерны, непрерывно зависят от точки $x \in \Lambda$ и дифференцируемо зависят от $x$ вдоль траекторий. Кроме того, сушествуют такие константы $c_{1}>0, \gamma_{1}>0, c_{2}>0, \gamma_{2}>0$, что

а) для любой точки $x \in \Lambda$, любого вектора $q \in E_{x}^{u *}$ и любого $t>0$, для которого $\Phi_{t}$ определено, справедливо неравенство $\left|q d \Phi_{t}^{*}\right|>c_{1} e^{\gamma_{1} t}|q|$;

b) для любой точки $x \in \Lambda$, любых векторов $v_{1}, v_{2}$, ортогональных пространству $E_{x}^{u *}$, и любого $t>0$, для которого $\Phi_{t}$ определено, справедливо неравенство

$$
S\left(d \Phi_{t} v_{1}, d \Phi_{t} v_{2}\right)>c_{2} e^{\gamma_{2} t} S\left(v_{1}, v_{2}\right)
$$

где $S\left(v_{1}, v_{2}\right)$ - площадь параллелограмма, порожденного векторами $v_{1}, v_{2}$.

Если выполнено условие $\left(\mathrm{H}^{*} \mathrm{U}\right)$, то пространства $E_{x}^{u *}$ строятся следующим стандартным образом.

Пусть $x(t)$ - траектория, $x(0)=x$. Если $x \in \Lambda$, то траектория $x(t)$ определена и принадлежит области $U$ для всех $t \in \mathbb{R}^{1}$. Из $\left(\mathrm{H}^{*} \mathrm{U}\right)$ следует, что

$$
\lim _{t \rightarrow-\infty} \mathscr{K}_{x(t)}^{u *} d \Phi_{-t}^{*}(x(t))
$$

сушествует и является одномерным пространством, которое и является пространством $E_{x}^{u *}$.

Лемма 3.3. Пусть выполнено условие $\left(\mathrm{H}^{*} \mathrm{U}\right)$. Тогда для любой точки $x \in \Lambda$ вектор $X(x)$ ортогонален пространству $E_{x}^{u *}$.

ДокАЗАТЕЛЬСтво. Пусть $x(t)$ - траектория точки $x, x(0)=x$. Так как $x \in \Lambda$, то траектория определена при всех $t \in \mathbb{R}^{1}$. Пространство $E_{x}^{u *}$ определено как предел $\lim _{t_{1} \rightarrow-\infty} \mathscr{K}_{x\left(t_{1}\right)}^{u *} d \Phi_{\left(-t_{1}\right)}^{*}$. Но $X(x(t))$ является решением уравнения в вариациях. Рассмотрим решение $q(t)$ сопряженного уравнения в вариациях с таким начальным условием, что $q(0) \in E_{x(0)}^{u *},|q(0)|=1$. В силу сохранения скалярного произведения получаем, что $(q(0), X(x(0)))=(q(t), X(x(t)))$, а в силу свойства а) в определении сингулярно гиперболической структуры получаем, что при $t<0$

$$
|q(0)|>c_{1} \exp \left(\gamma_{1}|t|\right)|q(t)|
$$


благодаря чему $\lim _{t \rightarrow-\infty}|q(t)|=0$. В силу ограниченности вектора $X(x)$ в области $U$ величина $(q(t), X(x(t)))$ стремится к нулю при $t \rightarrow-\infty$. Следовательно, $(q(0), X(x(0)))=0$. Лемма доказана.

В связи с последней леммой сформулируем еще один вариант условия сингулярной гиперболичности. Обозначим через $\Pi_{x}^{*}$ подпространство пространства $T_{x}^{*} U$, ортогональное вектору $X(x)$. Для неподвижной точки $x$ пространство $\Pi_{x}^{*}$ совпадает с $T_{x}^{*} U$.

УСловие $\left(\mathrm{H}^{*} \Pi\right)$. Для каждой точки $x \in U$ определен конус $\mathscr{K}_{x}^{u *} \subset \Pi_{x}^{*}$ с одномерным осевым пространством $Q_{x}^{*}$; семейство конусов инвариантно в том смысле, что существует такое $t_{1}>0$, что если $t>t_{1}$, то $\operatorname{clos}\left(\mathscr{K}_{x}^{u *} d \Phi_{t}^{*}\right) \subset \operatorname{int}\left(\mathscr{K}_{\Phi_{t}(x)}^{u *}\right)$. Кроме того, существуют такие константы $c_{1}>0, \gamma_{1}>0, c_{2}>0, \gamma_{2}>0$, что

а) для любой точки $x \in U$, любого вектора $q \in \mathscr{K}_{x}^{u *}$ и любого $t>0$, для которого $\Phi_{t}$ определено, справедливо неравенство $\left|q d \Phi_{t}^{*}\right|>c_{1} e^{\gamma_{1} t}|q|$;

b) для любой точки $x \in U$, любых векторов $v_{1}, v_{2}$, ортогональных некоторому вектору $q \in \mathscr{K}_{x}^{u *}$, и любого $t>0$, для которого $\Phi_{t}$ определено, справедливо неравенство

$$
S\left(d \Phi_{t} v_{1}, d \Phi_{t} v_{2}\right)>c_{2} e^{\gamma_{2} t} S\left(v_{1}, v_{2}\right)
$$

где $S\left(v_{1}, v_{2}\right)$ - плошадь параллелограмма, порожденного векторами $v_{1}, v_{2}$.

Вообще говоря, для произвольной системы приведенные условия $\left(\mathrm{H}^{*} \mathrm{U}\right),\left(\mathrm{H}^{*} \Pi\right)$, $\left(\mathrm{H}^{*} \Lambda\right)$ неэквивалентны. Для систем типа системы Лоренца условия $\left(\mathrm{H}^{*} \mathrm{U}\right),\left(\mathrm{H}^{*} \Pi\right)$ эквивалентны, а условие $\left(\mathrm{H}^{*} \Lambda\right)$ следует из условия $\left(\mathrm{H}^{*} \mathrm{U}\right)$. Доказательство эквивалентности мы приводить не будем. Остается неясньп, эквивалентны ли условия $\left(\mathrm{H}^{*} \mathrm{U}\right),\left(\mathrm{H}^{*} \Lambda\right)$ для систем типа системы Лоренца.

3.3. Неподвижные точки. Определим возможные типы неподвижных точек сингулярно гиперболических систем (не обязательно систем типа системы Лоренца).

Лемма 3.4. Пусть система $\dot{x}=X(x)$ в области $U$ удовлетворяет условию $\left(\mathrm{H}^{*} \mathrm{U}\right)$, матрица $D\left(x_{0}\right)$ неподвижной точки $x_{0}$ не имеет собственных значений с нулевой вещественной частью. Тогда неподвижная точка имеет тип $(L)$ или $(F)$. Если неподвижная точка $x_{0}$ имеет тип $(F)$, то существует окрестность этой точки, не содержащая точек множества $\Lambda$, кроме точки $x_{0}$ и ее неустойчивого многообразия.

ДокАЗАТЕЛЬСтво. Если существует инвариантное пространство $E_{x_{0}}^{*}$, то оно порождено левым собственным вектором $q_{1}$ матрицы $\left(-D\left(x_{0}\right)\right)$ с вещественным собственным значением. Обозначим это собственное значение через $\left(-\lambda_{1}\right)$. Матрица $D\left(x_{0}\right)$ имеет собственное значение $\lambda_{1}$ и тот же левый собственный вектор. Решение сопряженного уравнения в вариациях вдоль траектории $x(t) \equiv x_{0}$ имеет вид $q(t)=e^{-\lambda_{1} t} q_{1}$. Из п. а) условия ( $\left.\mathrm{H}^{*} \mathrm{U}\right)$ следует, что $-\lambda_{1}>0$; следовательно, $\lambda_{1}<0$.

Если существует конус $\mathscr{K}_{x_{0}}^{u *}$, содержащий пространство $E_{x_{0}}^{*}$, такой, что $\bigcap_{t>0} \mathscr{K}_{x_{0}}^{u *} d \Phi_{t}^{*}=E_{x_{0}}^{*}$, то собственное значение $\left(-\lambda_{1}\right)$ для матрицы $\left(-D\left(x_{0}\right)\right)$ имеет максимальную вешественную часть (это следует из описания траекторий 
в окрестности неподвижной точки линейной системы). Следовательно, для других собственных значений $-\lambda_{2},-\lambda_{3}$ матрицы $\left(-D\left(x_{0}\right)\right)$ справедливы неравенства $-\operatorname{Re} \lambda_{2}<-\lambda_{1},-\operatorname{Re} \lambda_{3}<-\lambda_{1}$, откуда следует, что $\operatorname{Re} \lambda_{2}>\lambda_{1}, \operatorname{Re} \lambda_{3}>\lambda_{1}$.

Очевидно, что решение уравнения в вариациях вдоль траектории $x(t) \equiv x_{0}$ имеет вид $\dot{v}=D\left(x_{0}\right) v$. Из соотношений ортогональности для левых и правых собственных векторов матрицы $D\left(x_{0}\right)$ следует, что инвариантное пространство $E \subset T_{x_{0}} U$, соответствуюшее собственньм значениям $\lambda_{2}, \lambda_{3}$, ортогонально вектору $q_{1}$. Нетрудно показать, что если $v_{1}(t), v_{2}(t)$ - решения уравнения $\dot{v}=D\left(x_{0}\right) v$, принадлежащие пространству $E, S(t)$ - площадь параллелограмма, порожденного векторами $v_{1}(t), v_{2}(t)$, то

$$
\frac{d S(t)}{d t}=\left(\operatorname{Re} \lambda_{2}+\operatorname{Re} \lambda_{3}\right) S(t) .
$$

Из условия b) в определении сингулярной гиперболичности следует, что $\operatorname{Re} \lambda_{2}+$ $\operatorname{Re} \lambda_{3}>0$.

Далее возможны следующие случаи:

1) $\lambda_{2}, \lambda_{3}$ - пара комплексно-сопряженных значений; тогда $\operatorname{Re} \lambda_{2}=\operatorname{Re} \lambda_{3}>0$ и неподвижная точка имеет тип $(F)$;

2) $\lambda_{2}, \lambda_{3}$ вешественные, $\lambda_{2} \leqslant \lambda_{3}$; тогда либо $\lambda_{2}>0$ и неподвижная точка имеет тип $(F)$; либо $\lambda_{2}<0$, тогда в силу неравенства $\lambda_{2}+\lambda_{3}>0$ справедливо $\lambda_{3}>0$ и неподвижная точка имеет тип $(L)$.

Докажем вторую часть леммы. Предположим, что неподвижная точка имеет тип $(F)$. Тогда для точки $x_{0}$ существуют одномерное устойчивое и двумерное неустойчивое многообразия. Как мы видели выше, сушествует система координат $\left(y_{1}, y_{2}, y_{3}\right)$, в которой устойчивое многообразие определяется уравнениями $y_{2}=0$, $y_{3}=0$, неустойчивое многообразие определяется уравнением $y_{1}=0$. Система уравнений в этой системе координат в случае вещественных корней имеет вид $\dot{y}_{j}=\lambda_{j} y_{j}+g_{j}(y), j=1,2,3$, в случае пары комплексно-сопряженных корней имеет вид $\dot{y}_{1}=\lambda_{1} y_{1}+g_{1}\left(y_{1}, z\right), \dot{z}=\lambda_{2} z+g_{2}\left(y_{1}, z\right)$, где $z=y_{2}+i y_{3}$. Здесь $g_{j}(y)$, $g_{j}(y, z)$ - такие функции, что их разложение в ряд Тейлора начинается с членов второго порядка малости. Специальная окрестность $V_{\delta, r}$ определяется неравенствами $\left|y_{1}\right|<\delta, y_{2}^{2}+y_{3}^{2}<r^{2}$. Если $r, \delta$ достаточно малы, то в эту окрестность траектории входят через множества $\left|y_{1}\right|=\delta, y_{2}^{2}+y_{3}^{2} \leqslant r^{2}$, а выходят из этой окрестности через множества $y_{2}^{2}+y_{3}^{2}=r^{2},\left|y_{1}\right|<\delta$.

Пусть $e_{1}-$ собственный вектор матрицы $D\left(x_{0}\right)$, соответствующий собственному значению $\lambda_{1}$. Тогда $\left(q_{1}, e_{1}\right) \neq 0$. Следовательно, в достаточно малой окрестности для вектора $w$, касательного к устойчивому многообразию, верно $\left(q_{1}, w\right) \neq 0$. Поэтому и для вектора $X(x)$, где $x$ принадлежит множествам $y_{2}^{2}+y_{3}^{2} \leqslant r^{2}, y_{1}= \pm \delta$ (обозначим эти множества $K_{+}, K_{-}$), при соответствующем выборе достаточно малых $r, \delta$ получаем $\left(q_{1}, X(x)\right) \neq 0$. В силу непрерывной зависимости пространства $E_{x}^{u *}$ от $x$ при достаточно малом $r$ имеем $(q, X(x)) \neq 0$ для $x \in K_{ \pm}, q \in E_{x}^{u *}$. Таким образом, точки $x \in K_{ \pm}$не принадлежат множеству $\Lambda$, так как для точек $x \in \Lambda$ в силу леммы $3.3(q, X(x))=0$. Следовательно, траектории всех точек $x \in K_{ \pm}$не принадлежат множеству $\Lambda$. Но траектория всякой точки $x \in V_{\delta, r}$, кроме точек неустойчивого многообразия, пересекается с множествами $K_{ \pm}$, и поэтому аттрактору могут принадлежать только точки, принадлежащие неустойчивому многообразию точки $x_{0}$. Лемма доказана. 
3.4. Ориентируемость и неориентируемость. Множество $\Lambda$ мы будем называть ориентируемым, если сушествует инвариантное векторное поле $p(x) \in$ $E_{x}^{u *}$, непрерывно зависяшее от точки $x \in \Lambda$ и дифференцируемое вдоль траекторий. Если аттрактор не является ориентируемым, то он называется неориентируемим.

Отметим, что в любом случае вектор $p(x)$ такой, что $p(x) \in E_{x}^{u *},|p(x)|=1$, в некоторой достаточно малой окрестности является непрерывным и дифференцируемьм вдоль траекторий. Поэтому в случае неориентируемого аттрактора существует инвариантная двузначная функция со значениями в $E_{x}^{u *}$, которая принимает значения $p(x),-p(x)$.

Двузначную функцию на $\Lambda$ со значениями в пространстве $T_{x}^{*} U$ будем называть двузначныцм векторным полем.

3.5. Функции $\lambda(x), \rho(x)$. Далее мы будем предполагать, что выполнено условие $\left(\mathrm{H}^{*} \Lambda\right)$.

Обозначим через $\lambda(x)$ функцию, определенную на $\Lambda$ равенством

$$
\frac{d|q(t)|}{d t}=\lambda(x(t))|q(t)|
$$

где $q(t)$ - решение сопряженного уравнения в вариациях вдоль траектории $x(t)$, $x(t) \in \Lambda, q(t) \in E_{x(t)}^{u *}$. Отметим, что из инвариантности семейства пространств $E_{x}^{u *}$ следует, что если $q(0) \in E_{x(0)}^{u *}$, то $q(t) \in E_{x(t)}^{u *}$ при любом $t \in \mathbb{R}^{1}$.

Пусть траектория $x(t)$ принадлежит множеству $\Lambda, v_{1}(t), v_{2}(t)$ - два линейно независимых решения уравнения в вариациях вдоль траектории $x(t)$, ортогональных пространству $E_{x(t)}^{u *}$. Через $\rho(x)$ обозначим функцию на $\Lambda$, определяемую равенством

$$
\frac{d S(t)}{d t}=\rho(x(t)) S(t)
$$

где $S(t)$ - плошадь параллелограмма, порожденного векторами $v_{1}(t), v_{2}(t)$. Отметим, что из леммы 3.1 следует, что если $v_{1}(0), v_{2}(0)$ ортогональны пространству $E_{x(0)}^{u *}$, то $v_{1}(t), v_{2}(t)$ ортогональны пространствам $E_{x(t)}^{u *}$ при любом $t \in \mathbb{R}^{1}$.

Несмотря на то что функции $\lambda(x), \rho(x)$ строились с помощью выбора решений $q(t), v_{1}(t), v_{2}(t)$, они не зависят от выбора этих решений, а зависят только от пространств, порожденных векторами $q(t)$ и соответственно $v_{1}(t), v_{2}(t)$.

Смысл функции $\lambda(x)$ - скорость роста решения сопряженного уравнения в вариациях в точке $x$, смысл функции $\rho(x)$ - скорость роста плошади параллелограмма, построенного по двум решениям уравнения в вариациях, ортогональным пространству $E_{x}^{u *}$, вдоль траектории, проходяшей через данную точку.

Если $x_{0}$ - неподвижная точка типа $(L)$ и $\lambda_{1}<\lambda_{2}<0<\lambda_{3}$ - собственные числа матрицы $D\left(x_{0}\right)$, то, как мы видели вьше, пространство $E_{x}^{u *}$ порождено левым собственным вектором матрицы $D\left(x_{0}\right)$, соответствуюшим собственному значению $\lambda_{1}$; нетрудно показать, что $\lambda\left(x_{0}\right)=-\lambda_{1}, \rho\left(x_{0}\right)=\lambda_{2}+\lambda_{3}$.

Лемма 3.5. Пусть $p(x) \in E_{x}^{u *}-$ непрерывное векторное поле на множестве $\Lambda$, дифференцируемое вдоль траекторий (однозначное для ориентируемого и двузначное для неориентируемого множества $\Lambda)$. Тогда существует такая функиия $\widehat{\lambda}(x)$, определенная на $\Lambda$, что пара $(p(x), \widehat{\lambda}(x))$ является 
решением уравнения

$$
\dot{p}(x)=-p(x) D(x)-\widehat{\lambda}(x) p(x) .
$$

Если $|p(x)|$ - постоянная величина, то $\widehat{\lambda}(x)=\lambda(x)$.

ДокАЗАтЕльство. Пусть $x(t)$ - траектория, $x(0)=x \in \Lambda, q(t)$ - решение сопряженного уравнения в вариациях вдоль траектории $x(t), p(x)=q(0)$. В силу инвариантности семейства $E_{x}^{u *}$ и одномерности пространств $E_{x}^{u *}$ имеем $p(x(t))=$ $a(t) q(t)$ с некоторой функцией $a(t)$. Тогда

$$
\begin{aligned}
\dot{p}(x(t)) & =a(t) \dot{q}(t)+\dot{a}(t) q(t)=-a(t) q(t) D(x(t))+\frac{\dot{a}(t)}{a(t)} a(t) q(t) \\
& =-p(x(t)) D(x(t))+\frac{\dot{a}(t)}{a(t)} p(x(t))
\end{aligned}
$$

откуда следует равенство (9) с функцией

$$
\widehat{\lambda}(x(t))=-\frac{\dot{a}(t)}{a(t)}=-\frac{d}{d t} \ln a(t) .
$$

Тем самым первое утверждение леммы доказано.

Докажем второе утверждение леммы.

Если $|p(x)|=c=$ const, то $a(t)=c /|q(t)|$, и в этом случае

$$
\widehat{\lambda}(x(t))=-\frac{d}{d t} \ln a(t)=\frac{d}{d t} \ln |q(t)|=\frac{1}{|q(t)|} \frac{d|q(t)|}{d t} .
$$

Сравнивая полученное равенство с (7), имеем $\widehat{\lambda}=\lambda$. Лемма доказана.

Обозначим через $q^{T}$ вектор-столбец, который получается из вектор-строки $q$ транспонированием. Очевидно, что $|q|=\sqrt{\left(q, q^{T}\right)}$.

Покажем, что если пара $(p(x), \widehat{\lambda}(x))$ - решение уравнения $(9)$, то для функции $\widehat{\lambda}(x)$ справедливо равенство

$$
\widehat{\lambda}(x)=-\frac{1}{|p|^{2}}\left(p D(x) p^{T}+\frac{1}{2} \frac{d}{d t}|p|^{2}\right) .
$$

Действительно,

$$
\begin{aligned}
\frac{d}{d t}|p|^{2} & =\left(\dot{p}, p^{T}\right)+\left(p, \dot{p}^{T}\right)=-\left(p D(t), p^{T}\right)-\widehat{\lambda}\left(p, p^{T}\right)-\left(p, D^{T} p^{T}\right)-\widehat{\lambda}\left(p, p^{T}\right) \\
& =-p\left(D(t)+D^{T}(t)\right) p^{T}-2 \widehat{\lambda}\left(p, p^{T}\right)
\end{aligned}
$$

откуда

$$
\widehat{\lambda}(x)=-\frac{1}{2|p|^{2}}\left(\frac{d}{d t}|p|^{2}+p\left(D(x)+D^{T}(x)\right) p^{T}\right) .
$$

Учитывая очевидное равенство $\left(p D(x), p^{T}\right)=\left(p, D^{T}(x) p^{T}\right)$, получаем соотношение (10). 
Заметим, что из (10) следует, что для любого вектора $p \in E_{x}^{u *}$ имеет место равенство

$$
\lambda(x)=-\frac{1}{|p|^{2}} p D(x(t)) p^{T} .
$$

Напомним, что две функции $h(x), \widetilde{h}(x)$ назьваются гомологичныцми, если существует такая непрерьвная функция $b(x)$, дифференцируемая вдоль траекторий, что $h(x)-\widetilde{h}(x)=d b(x) / d t$.

Нетрудно видеть, что если $h(x), \widetilde{h}(x)$ гомологичны, то для любой траектории величина $H=\int_{0}^{T}(h(x(t))-\widetilde{h}(x(t))) d t$ ограничена.

Лемма 3.6. Пусть пара $(p(x), \widehat{\lambda}(x))$ - решение уравнения $(9), p(x) \in E_{x}^{u *}$ для любой точки $x \in \Lambda$. Тогда для другой функиии $\widetilde{\widehat{\lambda}}(x)$ найдется векторное поле $\widetilde{p}(x)$ такое, что пара $(\widetilde{p}(x), \widetilde{\widehat{\lambda}}(x))$ является решением уравнения (9) в том и только том случае, когда функиии $\widehat{\lambda}(x), \widetilde{\widehat{\lambda}}(x)$ гомологичнь.

ДокаЗАтельство. Заметим, что если $p(x), \widetilde{p}(x) \in E_{x}^{u *}$, то $\widetilde{p}(x)=b(x) p(x)$ с некоторой функцией $b(x)$. Следовательно, $\dot{\tilde{p}}(x)=\dot{b}(x) p(x)+b(x) \dot{p}(x)$.

Учитьвая, что пары $(p(x), \widehat{\lambda}(x)),(\widetilde{p}(x), \widetilde{\widehat{\lambda}}(x))$ являются решениями $(9)$, получаeM

$$
\dot{\tilde{p}}(x)=-\widetilde{p}(x) D(x)-\widetilde{\widehat{\lambda}}(x) \widetilde{p}(x)=-b(x) p(x) D(x)-\widetilde{\widehat{\lambda}}(x) b(x) p(x) .
$$

С другой стороны,

$\dot{\tilde{p}}(x)=\frac{d}{d t} b(x) p(x)=\dot{b}(x) p(x)+b(x) \dot{p}(x)=\dot{b}(x) p(x)-b(x) p(x) D(x)-\widehat{\lambda}(x) b(x) p(x)$.

Из этих равенств следует, что $-\widetilde{\widehat{\lambda}}(x) b(x) p(x)=\dot{b}(x) p(x)-\widehat{\lambda}(x) b(x) p(x)$, откуда

$$
\widehat{\lambda}(x) b(x)-\widetilde{\widehat{\lambda}}(x) b(x)=\dot{b}(x), \quad \widehat{\lambda}(x)-\widetilde{\widehat{\lambda}}(x)=\frac{\dot{b}(x)}{b(x)}=\frac{d}{d t} \ln b(x),
$$

т.е. $\widehat{\lambda}(x), \widetilde{\widehat{\lambda}}(x)$ гомологичны.

Если $\widehat{\lambda}(x), \widetilde{\widehat{\lambda}}(x)$ гомологичны, функция $\widehat{\lambda}(x)$ соответствует векторному полю $p(x)$, то, как легко проверить, $\widetilde{\widehat{\lambda}}(x)$ соответствует векторному полю $\widetilde{p}(x)$, определяемому соотношением $\widetilde{p}(x)=e^{b(x)} p(x)$, где $b(x)$ - такая функция, что $\widehat{\lambda}(x)-$ $\widetilde{\widehat{\lambda}}(x)=d(\ln b(x)) / d t$.

Лемма доказана.

Лемма 3.7. Справедливо равенство

$$
\rho(x)=\lambda(x)+\operatorname{div} X(x) .
$$


ДоказАТЕЛЬСТво. Пусть $p(x)$ - векторное поле на $\Lambda$ (однозначное для ориентируемого случая и двузначное для неориентируемого случая) такое, что $p(x) \in$ $E_{x}^{u *},|p(x)|=1$. Тогда пара $(p(x), \lambda(x))$ является решением (9) (лемма 3.5 ).

Пусть $x(t)$ - траектория, $x(t) \in \Lambda, v_{2}(t), v_{3}(t)$ - два линейно независимых решения уравнения в вариациях вдоль траектории $x(t)$, ортогональных вектору $p(x(t)), S(t)$ - площадь параллелограмма, порожденного векторами $v_{2}(t), v_{3}(t)$. В ходе доказательства леммы мы будем обозначать матрицу $D(x(t))$ через $D(t)$ или $D$, функцию $\lambda(x(t))$ через $\lambda(t)$, вектор $p(x(t))$ через $p(t)$.

Пусть $v_{1}(t)$ - третье решение уравнения в вариациях, линейно не зависимое от $v_{2}(t), v_{3}(t)$.

Объем параллелепипеда, порожденного векторами $v_{1}, v_{2}, v_{3}$, можно вычислить из соотношения $V(t)=S(t)\left(p, v_{1}\right)$ (следует из равенства $|p(x)|=1$ ). Поэтому

$$
\frac{d}{d t} V(t)=\dot{S}(t)\left(p, v_{1}\right)+S(t) \frac{d}{d t}\left(p, v_{1}\right)=\dot{S}(t)\left(p, v_{1}\right)+S(t)\left(\dot{p}, v_{1}\right)+S(t)\left(p, \dot{v}_{1}\right) .
$$

Из равенств $\dot{v}_{1}=D(t) v_{1}, \dot{p}=-p D(t)-\lambda(t) p, \quad\left(p(t), D(t) v_{1}\right)=\left(p(t) D(t), v_{1}\right)$ следует, что

$$
\begin{aligned}
\frac{d}{d t} V(t) & =\dot{S}(t)\left(p, v_{1}\right)+S(t)\left(-p D(t), v_{1}\right)-\lambda(t) S(t)\left(p, v_{1}\right)+S(t)\left(p, D(t) v_{1}\right) \\
& =(\dot{S}(t)-\lambda(x(t)) S(t))\left(p, v_{1}\right) .
\end{aligned}
$$

С другой стороны, $\dot{V}=\operatorname{div} X \cdot V=\operatorname{div} X \cdot S \cdot\left(p, v_{1}\right)$ и потому

$$
(\dot{S}-\lambda(t) S)\left(p, v_{1}\right)=\operatorname{div} X \cdot S \cdot\left(p, v_{1}\right),
$$

откуда получаем $\dot{S}=(\operatorname{div} X+\lambda(t)) S$.

В то же время $\dot{S}(t)=\rho(t) S(t)$. Следовательно, $\rho(x)=\lambda(x)+\operatorname{div} X(x)$. Лемма доказана.

СлЕДСТВИЕ. Функция $\rho(x)$ не зависит от выбора векторов $v_{1}, v_{2}$, принадлежащих ортогональному дополнению к пространству $E_{x}^{u *}$.

Вычислим величину $\lambda(x)$ для гиперболического цикла. Предположим, что $x(t)-$ периодическая траектория периода $T$. Любая точка $x_{0}$ на этой траектории неподвижна относительно $\Phi_{T}$. Хорошо известно, что оператор $d \Phi_{T}$ в пространстве $T_{x_{0}} U$ имеет собственное значение 1 (оно соответствует вектору $X(x(0))$ ). Если цикл гиперболичен, то имеются еше два собственных значения $\mu_{1}, \mu_{2}$, причем одно из них меньше 1 (по модулю), другое больше 1 . Пусть $0<\left|\mu_{1}\right|<1<\left|\mu_{2}\right|$. Из этих неравенств видно, что $\mu_{1}, \mu_{2}$ не могут быть парой комплексно-сопряженных, поэтому они вешественные. Так как $\mu_{1} \mu_{2}>0$, то они либо оба положительны, либо оба отрицательны. В первом случае будем называть цикл ориентируемым, во втором - неориентируемым.

Предположим, что цикл ориентируемый. Тогда $0<\mu_{1}<1<\mu_{2}$. Величины $1 / \mu_{1}, 1,1 / \mu_{2}-$ собственные числа оператора $d \Phi_{T}^{*}$. Мы можемпостроить векторное поле для точек траектории $x(t)$, полагая $p(t)=q(t) e^{-\lambda_{1} t}$, где $q(t)$ - решение сопряженного уравнения в вариациях вдоль траектории $x(t), p(0)=q(0)$ - левый собственный вектор оператора $d \Phi_{T}^{*}$, соответствуюший собственному значению $1 / \mu_{1}$, 
$\lambda_{1}$ выбрано так, что $q(T) e^{-\lambda_{1} T}=q(0)$. Отсюда видно, что $e^{-\lambda_{1} T}=\mu_{1}$. Для функционала $p(t)$ справедливо соотношение

$$
\dot{p}(t)=-\lambda_{1} e^{-\lambda_{1} t} q_{1}+e^{-\lambda_{1} t} \dot{q}_{1}=-\widehat{\lambda} p(t)-e^{-\lambda_{1} t} q_{1} D(t)=-\lambda_{1} p(t)-p D(t),
$$

откуда видно, что $\lambda_{1}$ совпадает с $\hat{\lambda}$, определенным уравнением $(9)$ для поля $p(x)$.

В случае неориентируемого цикла следует рассмотреть решение $q(t)$ и выбрать $\lambda_{1}$ так, что $q(T) e^{-\lambda_{1} T}=-q(0)$. Тогда окажется, что вектор $p(t)$ определяет двузначное векторное поле на цикле.

Следовательно, для гиперболического цикла сушествует решение $(p(x), \widehat{\lambda}(x))$ уравнения (9), которое при отрицательных мультипликаторах является двузначной функцией со значениями $p(x),-p(x)$. При этом $\widehat{\lambda}(x)=-\frac{1}{T} \ln \left|\mu_{1}\right|$, а функция $\lambda(x)$ гомологична постоянной, равной $-\frac{1}{T} \ln \left|\mu_{1}\right|$.

В заключение настоящего параграфра отметим некоторые простые факты.

Следуюшее условие эквивалентно условиям a), b) условия $\left(\mathrm{H}^{*} \Lambda\right)$.

На $\Lambda$ существует инвариантное семейство одномерных подпространств $E_{x}^{u *} \subset T_{x}^{*} U$, непрерывно зависящих от $x$ и дифференцируемых вдоль траекторий. Кроме того, существуют константь $c_{1} \in \mathbb{R}^{1}, \gamma_{1}>0, c_{2} \in \mathbb{R}^{1}, \gamma_{2}>0$ такие, что для функиий $\lambda(x), \rho(x)$, построенных выше для семейства $E_{x}^{u *}$, и для любой траектории $x(t) \in \Lambda$ при любом $t>0$ справедливь неравенства

$$
\left.\left.\mathrm{a}^{\prime}\right) \quad \int_{0}^{t} \lambda(x(\tau)) d \tau>c_{1}+\gamma_{1} t, \quad \mathrm{~b}^{\prime}\right) \quad \int_{0}^{t} \rho(x(\tau)) d \tau>c_{2}+\gamma_{2} t
$$

Последнюю часть приведенного условия можно переформулировать следующим образом: существуют константы $T>0, k>0$ такие, что для любой траектории $x(t) \in \Lambda$ справедливы неравенства

$$
\left.\left.\mathrm{a}^{\prime \prime}\right) \quad \int_{0}^{T} \lambda(x(\tau)) d \tau>k, \quad \mathrm{~b}^{\prime \prime}\right) \quad \int_{0}^{T} \rho(x(\tau)) d \tau>k .
$$

Наконец, отметим, что величины

$$
\lim \sup _{T \rightarrow \infty}\left(-\frac{1}{T} \int_{0}^{T} \lambda(x(t)) d t\right), \quad \lim \sup _{T \rightarrow \infty}\left(\frac{1}{T} \int_{0}^{T} \rho(x(t)) d t\right)
$$

являются характеристическими показателями.

ЗАМЕчАНИЕ. С точки зрения вычислений на компьютере проверка условия $\left(\mathrm{H}^{*} \mathrm{U}\right)$ гораздо проще, чем вычисление классических условий равномерной гиперболичности. Предположим, что $\Lambda$ - аттрактор. Для проверки правдоподобия выполнения условия $\left(\mathrm{H}^{*} \mathrm{U}\right)$ следует вычислить достаточно длинную траекторию $x(t)$, которая заполняет аттрактор достаточно плотно, вдоль этой траектории вычислить поле $q(x) \in E_{x}^{u *}$ (в случае выполнения условия $\left(\mathrm{H}^{*} \mathrm{U}\right)$ вычисления устойчивы), вычислить функции $\lambda(x), \rho(x)$ по формулам $(10),(11)$ и убедиться в выполнении неравенств (12) или (13).

Вычислить вектор $q(x)$ несложно. Для этого можно взять произвольный вектор $q(0)$ и решить с ним сопряженное уравнение в вариациях. В случае сингулярно гиперболической системы пространство, порожденное вектором $q(x(t))$, сходится с экспоненциальной скоростьюк $E_{x}^{u *}$. 


\section{§4. Эквивалентность условий гиперболичности и сингулярной гиперболичности}

В настояшем параграфе мы предполагаем, что рассматриваемая система $\dot{x}=$ $X(x)$ удовлетворяет условиям (L1)-(L4). Отображение последования на поверхности $P$ в этом параграфе обозначается через $F$. Через $U$ обозначается окрестность, построенная выше (см. лемму 2.3), через $\Lambda$ - аттрактор системы.

ТЕОРема 4.1. Пусть выполнены условия (L1)-(L4) $и \operatorname{div} X(x)<0$. Тогда гиперболичность отображения $F$ әквивалентна сингулярной гиперболичносmu потока $\Phi_{t}$.

Доказательство теоремы разделим на две части. В первой части мы докажем гиперболичность отображения $F$, предполагая сингулярную гиперболичность потока $\Phi_{t}$. Во второй части докажем сингулярную гиперболичность потока, предполагая гиперболичность отображения $F$.

4.1. Вывод гиперболичности отображения $F$ из сингулярной гиперболичности потока. Предположим, что для потока $\Phi_{t}$ выполнено условие $\left(\mathrm{H}^{*} \mathrm{U}\right)$. Как мы видели выше, на $\Lambda$ сушествует семейство пространств $E_{x}^{u *}$, удовлетворяющее условию $\left(\mathrm{H}^{*} \Lambda\right)$. Для точек $x \in \Lambda \cap P$ обозначим через $E_{x}^{u}$ пространство, ортогональное пространству $E_{x}^{u *}$ и касательное к плоскости $P$. Из леммы 3.3 и из трансверсальности вектора $X(x)$ и поверхности $P$ следует, что касательная плоскость к поверхности $P$ не совпадает с плоскостью, ортогональной пространству $E_{x}^{u *}$. Отсюда и из соображений размерности следует, что пространство $E_{x}^{u}$ одномерно. Из непрерывности семейства пространств $E_{x}^{u *}$ следует, что семейство пространств $E_{x}^{u}$ непрерывно зависит от $x$ на $P$. Из инвариантности семейства $E_{x}^{u *}$ и леммы 3.1 следует, что семейство пространств $E_{x}^{u}$ инвариантно относительно $d F$.

Для точки $x \in P \cap \Lambda$ обозначим через $\mu(x)$ величину $|d F(v)| /|v|$, где $v \in E_{x}^{u}$. Так как пространство $E_{x}^{u}$ одномерно, то $\mu(x)$ не зависит от выбора вектора $v \in$ $E_{x}^{u}$. Величина $\mu(x)$ называется коэффициентом растяжения в неустойчивом направлении в точке $x$. Положим $\xi(x)=\ln \mu(x)$. Через $t(x)$ обозначим время возврашения на поверхность $P$, через $\eta(x)$ - величину $\eta(x)=\int_{0}^{t(x)} \rho(x(t)) d t$, через $H(x)$ - функцию $H(x)=\ln (|X(x)| \sin \beta(x))$, где $\beta(x)$ - угол между вектором $X(x)$ и пространством $E_{x}^{u}$.

Отметим, что функции $\mu(x), \xi(x), \eta(x)$ определены только для тех точек $x \in P$, положительные полутраектории которых пересекаются с $P$.

ЛЕмма 4.2. Справедливо равенство

$$
\eta(x)=\xi(x)+H(F(x))-H(x) .
$$

ДокАЗАТЕЛьство. Пусть $x \in \Lambda \cap P, v_{0} \in E_{x}^{u}, S_{0}$ - площадь параллелограмма, порожденного векторами $v_{0}$ и $X(x), v_{1}=d F\left(v_{0}\right), v_{2}=d \Phi_{t(x)}\left(v_{0}\right), S_{1}$ - площадь параллелограмма, порожденного векторами $v_{1}$ и $X(F(x))$. Так как $v_{1}=v_{2}+$ $\alpha X(F(x))$ с некоторой константой $\alpha \in \mathbb{R}^{1}$, то $S_{1}$ равна площади параллелограмма, порожденного векторами $v_{2}$ и $X(F(x))$. Поэтому

$$
S_{1}=\exp \left(\int_{0}^{t(x)} \rho(x(t)) d t\right) S_{0}=e^{\eta(x)} S_{0}
$$


С другой стороны,

$$
S_{0}=\left|v_{0}\right||X(x)| \sin \beta(x), \quad S_{1}=\left|v_{1}\right||X(F(x))| \sin (\beta(F(x))) .
$$

Следовательно,

$$
\left|v_{1}\right||X(F(x))| \sin (\beta(F(x)))=\left|v_{0}\right||X(x)| \sin (\beta(x)) e^{\eta(x)} .
$$

Поделив сначала обе части последнего равенства на $\left|v_{0}\right|$, а затем прологарифмировав их, с учетом определения функции $H(x)$ получим

$$
\ln \frac{\left|v_{1}\right|}{\left|v_{0}\right|}+H(F(x))=H(x)+\eta(x) .
$$

Но по определению $\xi(x)=\ln \left(\left|v_{1}\right| /\left|v_{0}\right|\right)$, откуда и получаем требуемое равенство. Лемма доказана.

Лемма 4.3. Пусть выполнено условие $\left(\mathrm{H}^{*} \Lambda\right)$. Тогда для построенного въцше семейства пространств $E_{x}^{u} \subset T_{x} P$ существуют такие константы $c_{0}>0, \mu_{0}>0$, что для любой точки $x \in \Lambda \cap P$, любого вектора $v_{0} \in \mathscr{K}_{x}^{u}$ и любого натурального $n$, для которого $F^{n}(x)$ определено, справедливо неравенство $\left|d F^{n}(v)\right|>c_{0} \mu_{0}^{n}|v|$.

ДокАЗАТЕЛЬСТво. Пусть $x \in \Lambda, x(t)$ - положительная полутраектория, $x(0)=x, v_{0} \in \mathscr{K}_{x}^{u}, v(t)$ - решение уравнения в вариациях вдоль траектории $x(t)$ с начальным условием $v(0)=v_{0}, t_{1}<t_{2}<t_{3}<\cdots$ - времена возврашения полутраектории $x(t)$ на поверхность $P, x_{n}=x\left(t_{n}\right), S_{n}$ - площадь параллелограмма, порожденного векторами $X\left(x_{n}\right)$ и $v\left(t_{n}\right), \widehat{S}_{n}$ - площадь параллелограмма, порожденного векторами $X\left(x_{n}\right)$ и $d F^{n}\left(v_{0}\right)$.

Нетрудно заметить, что $d F^{n}\left(v_{0}\right)=v\left(t_{n}\right)+\alpha_{n} X\left(x_{n}\right)$, где $\alpha_{n}$ - некоторый множитель. Площадь параллелограмма, порожденного векторами $X\left(x_{n}\right), v\left(t_{n}\right)$, совпадает с плошадью параллелограмма, порожденного векторами $X\left(x_{n}\right), v\left(t_{n}\right)+$ $\alpha_{n} X\left(x_{n}\right)$. Следовательно, $S_{n}=\widehat{S}_{n}$. В силу определения функции $\rho(x)$ (равенство (8)) и в силу равенства $S_{n}=\widehat{S}_{n}$ справедливо

$$
\widehat{S}_{n}=\widehat{S}_{0} \exp \left(\int_{0}^{t_{n}} \rho(t) d t\right) .
$$

Из $\left(\mathrm{H}^{*} \Lambda\right)$ следует, что $\widehat{S}_{n}>\widehat{S}_{0} c_{2} e^{\gamma_{2} t_{n}}$ (константы $c_{2}, \gamma_{2}$ определены в условии $\left.\left(\mathrm{H}^{*} \Lambda\right)\right)$. Так как время возвращения ограничено снизу, то $t_{n}>n T$, где $T-$ минимальное время возвращения на поверхность $P$, и потому $\widehat{S}_{n}>\widehat{S}_{0} c_{2} e^{n \gamma_{2} T}$ для констант $c_{2}, \gamma_{2}$. Следовательно, справедлива оценка $\widehat{S}_{n}>c_{2} \mu_{0}^{n} \widehat{S}_{0}$, где $\mu_{0}=e^{\gamma_{2} T}$. Отсюда получаем

$$
\left|d F^{n}(v)\right| H\left(x_{n}\right) \geqslant c_{2} \mu^{n}|v| H\left(x_{0}\right) .
$$

Но в силу ограниченности величины $H\left(x_{0}\right) / H\left(x_{n}\right)$

$$
\left|d F^{n}(v)\right| \geqslant c_{0} \mu_{0}^{n}|v|
$$

c некоторой константой $c_{0}>0$.

Лемма доказана.

Из только что доказанной леммы и леммы 2.7 следует справедливость следующего утверждения. 
СлЕДСТВИЕ. Если выполнено условие $\left(\mathrm{H}^{*} \Lambda\right)$ u $\operatorname{div} X(x)<0$, то для отображения $F$ и построенного семейства пространств $E_{x}^{u}$ выполнено условие $(\mathrm{H} \Lambda)$.

4.2. Вывод условия (HU) из условия $\left(\mathrm{H}^{*} \mathrm{U}\right)$. Предположим, что для потока выполнено условие $\left(\mathrm{H}^{*} \mathrm{U}\right)$. Как мы видели выше, пространство $E_{x}^{u *}$ ортогонально вектору $X(x)$, который предполагается трансверсальньм к поверхности $P$ (условие (L2)). Следовательно, если мы вместо конуса $\mathscr{K}_{x}^{u *}$ рассмотрим конус $\widetilde{\mathscr{K}}_{x}^{u *}=\mathscr{K}_{\Phi_{-t}(x)}^{u *} d \Phi_{t}$ для достаточно большого $t>0$, то семейство конусов $\widetilde{\mathscr{K}}_{x}^{u *}$ будет инвариантньм и для всякого вектора $q \in \widetilde{\mathscr{K}}_{x}^{u *}$ ортогональное дополнение к вектору $q$ в пространстве $T_{x} U$ не будет совпадать с $T_{x} P$. Из соображений размерности следует, что для каждого $q \in \widetilde{\mathscr{K}}_{x}^{u *}$ множество векторов $v \in T_{x} U$, касательных к $P$ и ортогональных вектору $q$, образует одномерное пространство. Обозначим это пространство $E_{q}$.

Обозначим через $\mathscr{K}_{x}^{u}$ объединение пространств $E_{q}$ по всем $q \in \widetilde{\mathscr{K}}_{x}^{u *}$. Очевидно, что множество $\mathscr{K}_{x}^{u}$ является конусом. Инвариантность и непрерывность семейства конусов $\mathscr{K}_{x}^{u}$ следует из инвариантности и непрерьвности семейства конусов $\widetilde{\mathscr{K}}_{x}^{u *}$.

Доказательство оценки $\left|d F_{p}^{n}(v)\right|>c \mu^{n}|v|$ для $v \in \mathscr{K}_{x}^{u}$ принципиально не отличается от доказательства леммы 4.3 , и поэтому мы его не приводим. Существование системы конусов $\mathscr{K}_{x}^{s}$ и условие (HU) следуют из леммы 2.7 .

Тем самьм доказательство условия (HU) закончено.

4.3. Вывод условия $\left(\mathrm{H}^{*} \Lambda\right)$ из гиперболичности отображения последования. Напомним, что собственные числа матрицы $D\left(x_{0}\right)$ неподвижной точки $x_{0}$ типа $(L)$ таковы, что $\lambda_{1}<\lambda_{2}<0<\lambda_{3}, \lambda_{2}+\lambda_{3}>0$.

Лемма 4.4. Пусть точка $x$ принадлежит множеству $W^{s}\left(x_{0}\right) \backslash W^{s s}\left(x_{0}\right)$, где $x_{0}$ - неподвижная точка типа $(L), x(t)$ - положительная полутраектория, $x(0)=x$. Справедливь следующие утверждения.

i) Вектор $e_{2}(t)=X(x(t)) /|X(x(t))|$ при $t \rightarrow+\infty$ сходится $\kappa$ собственному вектору, соответствующему собственному значению $\lambda_{2}$.

ii) Если вектор $v \in T_{x} U$ трансверсален многообразию $W^{s}\left(x_{0}\right), v(t)$ решение уравнения в вариациях, соответствующее полутраектории $x(t)$, $v(0)=v$, mо $v(t) /|v(t)|$ при $t \rightarrow+\infty$ сходится $к$ собственному вектору, соответствующему собственному значению $\lambda_{3}$.

iii) Если $q \in T_{x}^{*} U-$ функционал, ортогональный вектору $X(x)$ и одному из векторов $v \in T_{x} U$, трансверсальному многообразию $W^{s}\left(x_{0}\right), q(t)$ - решение сопряженного уравнения в вариачиях с начальным условием $q(0)=q$, то $p(t)=q(t) /|q(t)|$ при $t \rightarrow+\infty$ сходится $\kappa$ левому собственному вектору матрици $D\left(x_{0}\right)$, соответствующему собственному значению $\lambda_{1}$.

ДокАЗАТЕЛЬСтво. Заметим, что сужение системы на $W^{s}\left(x_{0}\right)$ имеет неподвижную точку типа узел с собственными числами $\lambda_{1}<\lambda_{2}<0$. Поэтому утверждение i) следует из описания траекторий двумерных систем в окрестности неподвижной точки типа узел.

Утверждение іi) мы докажем для случая, когда система в окрестности точки $x_{0}$ приводится гладким преобразованием к линейной. В соответствуюшей системе 
координат $\left(y_{1}, y_{2}, y_{3}\right)$ система уравнений $\dot{x}=X(x)$ имеет вид

$$
\dot{y}_{1}=\lambda_{1} y_{1}, \quad \dot{y}_{2}=\lambda_{2} y_{2}, \quad \dot{y}_{3}=\lambda_{3} y_{3}
$$

Точно такой же вид имеет система уравнений в вариациях. Если в этой системе координат $v=\left(y_{1}(0), y_{2}(0), y_{3}(0)\right)$, то из трансверсальности $v$ и $W^{s}\left(x_{0}\right)$ следует, что $y_{3}(0) \neq 0$. Решение системы $(14)$ имеет вид

$$
y_{1}(t)=e^{\lambda_{1} t} y_{1}(0), \quad y_{2}(t)=e^{\lambda_{2} t} y_{2}(0), \quad y_{3}(t)=e^{\lambda_{3} t} y_{3}(0)
$$

Учитывая, что $y_{3}(0) \neq 0$, и соотношения $\lambda_{1}<\lambda_{2}<0<\lambda_{3}$, получаем, что $y_{3} \rightarrow \infty$, $y_{1} \rightarrow 0, y_{2} \rightarrow 0$ при $t \rightarrow+\infty$. Отсюда следует ii).

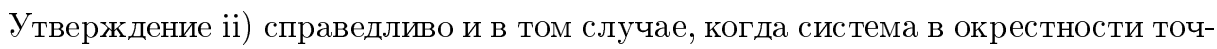
ки $x_{0}$ не приводится гладким преобразованием к линейной . Однако мы этот случай оставим без доказательства.

Утверждение іiі) доказывается из соотношения ортогональности. Так как $q(t)$ остается ортогональным вектору $X(x(t))$ и вектору $v(t)$, построенному при доказательстве ii) (см. лемму 3.1), то из і) и іi) следует, что пределом функционала $p(t)$ является функционал, ортогональный собственньм векторам матрицы $D\left(x_{0}\right)$, соответствующим собственным значениям $\lambda_{2}$ и $\lambda_{3}$. Но это и есть левый собственный вектор, соответствующий собственному значению $\lambda_{1}$. Лемма доказана.

Лемма 4.5. Пусть на $P \cap \Lambda$ определено семейство неустойчивых пространств $E_{x}^{u}$, удовлетворяющее условию $(\mathrm{H} \Lambda)$. Тогда на $\Lambda$ существует семейство пространств $E_{x}^{u *} \subset T_{x}^{*}$; семейство $E_{x}^{u *}$ инвариантно относительно $d \Phi_{t}^{*}$, зависимость пространств $E_{x}^{u *}$ от $x$ является непрерывной и дифферениируемой вдоль траекторий.

ЗАмечАниЕ. Данная лемма не утверждает выполнения условия $\left(\mathrm{H}^{*} \Lambda\right)$, так как в ней не утверждается выполнение свойств а) и b) из условия $\left(\mathrm{H}^{*} \Lambda\right)$.

ДокАЗАТЕЛьСтво. Построим пространства $E_{x}^{u *}$ для различных точек аттрактора.

Для неподвижных точек типа $(L)$ определим $E_{x}^{u *}$ как пространство, порожденное левым собственным вектором, соответствующим минимальному (отрицательному) собственному значению.

Далее определим пространство $E_{x}^{u *}$ для точек многообразия $W^{u}\left(x_{j}\right)$ неподвижной точки $x_{j}$ типа $(L)$.

Сначала отметим, что для неподвижной точки $x_{j}$ типа $(L)$ имеется конус $\mathscr{K}_{x_{j}}^{u *} \subset$ $T_{x_{j}}^{*} U$ с осевым пространством $E_{x_{j}}^{u *}$, который сжимается под действием $d \Phi_{t}^{*}$. Очевидно, что этим же свойством обладает тот же конус для точек, достаточно близких к $x_{j}$. Поэтому мы можем определить $E_{x}^{u *}$ для $x \in W^{u}\left(x_{j}\right)$ как предел

$$
E_{x}^{u *}=\lim _{t \rightarrow \infty} E_{x_{j}}^{u *} d \Phi_{t}^{*}\left(\Phi_{-t}(x)\right)
$$

Для точек $x \in \Lambda \cap P$ определим $E_{x}^{u *}$ как пространство, ортогональное вектору скорости и пространству $E_{x}^{u}$. 
Для траекторий точек $x \in \Lambda \cap P$ определим пространства $E_{x(t)}^{u *}$ из условия инвариантности. Для этого построим решение $q(t)$ сопряженного уравнения в вариациях вдоль траектории $x(t), x(0) \in \Lambda \cap P, q(0) \in E_{x(0)}^{u *}$, и определим пространство $E_{x(t)}^{u *}$ как пространство, порожденное вектором $q(t)$.

Из доказательства леммы 2.3 видно, что все точки аттрактора $\Lambda$ исчерпываются траекториями точек $\Lambda \cap P$, неподвижными точками типа $(L)$ и их неустойчивыми многообразиями. Тем самым пространства $E_{x}^{u *}$ построены для всех точек аттрактора.

Инвариантность пространств $E_{x}^{u *}$ следует из инвариантности пространств $E_{x}^{u}$ относительно $d F$.

Действительно, единственное место, где могут возникнуть сомнения, - это пересечение неустойчивого многообразия неподвижной точки типа $(L)$ с поверхностью $P$ и при возврашении траектории на поверхность $P$.

Пусть $x(t)$ - траектория, $x(0) \in P \cap \Lambda, x\left(t_{1}\right) \in P \cap \Lambda, v(t)$ - решение уравнения в вариациях вдоль $x(t), q(t)$ - решение сопряженного уравнения в вариациях вдоль $x(t), v(0) \in E_{x(0)}^{u}, q(0) \in E_{x(0)}^{u *}$. В силу леммы $3.3 q\left(t_{1}\right)$ ортогонально вектору $X\left(x_{1}\right)$ и вектору $v\left(t_{1}\right)$. Но пространство $E_{x}^{u}$ порождено вектором $w=d F(v(0))$, равным $v\left(t_{1}\right)+\alpha X\left(x\left(t_{1}\right)\right)$ с некоторьм коэффициентом $\alpha$. Следовательно, $q\left(t_{1}\right)$ ортогонально вектору $X\left(x_{1}\right)$ и вектору $d F(v)$, который в силу инвариантности лежит в $E_{x\left(t_{1}\right)}^{u}$ (и порождает $\left.E_{x\left(t_{1}\right)}^{u}\right)$, т.е. $q\left(t_{1}\right) \in E_{x\left(t_{1}\right)}^{u *}$.

Докажем непрерывность семейства $E_{x}^{u *}$. Сначала докажем непрерывность семейства в неподвижной точке $x_{j}$ типа $(L)$.

Пусть $x_{j}$ - неподвижная точка типа $(L), x \in W^{s}\left(x_{j}\right)$. Из трансверсальности пространства $E_{x}^{u}$ и многообразия $W^{s}\left(x_{j}\right)$ следует, что пространство $E_{x}^{u *}$ не ортогонально плоскости, касательной к многообразию $W^{s}\left(x_{j}\right)$. В силу леммы 4.4 для траектории $x(t)$, лежащей в $W^{s}\left(x_{j}\right)$, пространство $E_{x(t)}^{u *}$ сходится к пространству $E_{x_{j}}^{u *}$. Непрерывность в остальных точках очевидна. Лемма доказана.

В силу только что доказанной леммы для точек $x \in \Lambda$ сушествует инвариантное семейство пространств $E_{x}^{u *} \subset T_{x}^{*} U$. Как и вьше, по семейству $E_{x}^{u *}$ определим функции $\lambda(x), \rho(x)$.

Лемма 4.6. Пусть на $\Lambda \cap P$ выполнено условие (НА). Тогда существуют такие константы $T>0, k>0$, что для семейства $E_{x}^{u *}$, построенного в предъдущей лемме, $\int_{0}^{T} \rho(x(t)) d t>k$ для всякой траектории $x(t) \in \Lambda$.

ДоКАЗАТЕЛЬСТво. Заметим, что для неподвижной точки $x_{j}$ типа $(L) \rho\left(x_{j}\right)=$ $\lambda_{2}+\lambda_{3}>0$. Следовательно, сушествует такая окрестность $V_{j}$ точки $x_{j}$, что в этой окрестности $\rho(x)>\theta$ для некоторой константы $\theta>0$. Пусть $\nu>0$ - такая константа, что $\rho(x)>-\nu$ для любой точки $x \in \Lambda$.

Сушествует такое $T_{1}>0$, что положительная полутраектория каждой точки $x \in \Lambda$ до пересечения с поверхностью $P$ проводит вне окрестностей $V_{j}$ время, не большее чем $T_{1}$. Отсюда следует, что если траектория $x(t)$ не имеет точек пересе- 
чения с поверхностью $P$ для $t \in\left(t_{1}, t_{2}\right)$, то

$$
\begin{array}{ll}
\int_{t_{1}}^{t_{2}} \rho(t) d t \geqslant-\left(t_{2}-t_{1}\right) \nu \geqslant-T_{1} \nu, & t_{2}-t_{1} \leqslant T_{1}, \\
\int_{t_{1}}^{t_{2}} \rho(t) d t \geqslant\left(t_{2}-t_{1}-T_{1}\right) \theta-T_{1} \nu \geqslant-T_{1} \nu, \quad t_{2}-t_{1}>T_{1} .
\end{array}
$$

Пусть $k>0$ - произвольное число. В силу гиперболичности отображения $F$ и леммы 4.2 существует такое $n_{0}$, что для каждой точки $x \in \Lambda \cap P$ справедливо неравенство

$$
\eta(x)+\eta(F(x))+\cdots+\eta\left(F^{n_{0}-1}(x)\right)>2 T_{1} \nu+k,
$$

где $\eta(x)=\int_{0}^{t(x)} \rho(\tau) d \tau($ здесь $t(x)$ - время возвращения точки $x$ на поверхность $P$ ).

Пусть $T$ настолько велико, что

$$
\theta\left(T-\left(n_{0}+2\right) T_{1}\right)-\nu\left(n_{0}+2\right) T_{1}>k .
$$

Пусть $x(t)$ - отрезок траектории точки $x \in \Lambda, t \in[0, T], 0 \leqslant t_{0}<t_{1}<t_{2}<$ $\cdots<t_{n} \leqslant T$ - времена попадания отрезка траектории $x(t)$ на поверхность $P$.

Рассмотрим два случая.

Первый случай $\left(n>n_{0}\right)$. В этом случае по определению величины $\eta(x)$

$$
\int_{t_{0}}^{t_{n}} \rho(x(t)) d t=\eta\left(x\left(t_{0}\right)\right)+\cdots+\eta\left(x\left(t_{n-1}\right)\right)>k+2 T_{1} \nu
$$
Из (15) и (16) следует, что $\int_{0}^{t_{0}} \rho(x(t)) d t \geqslant-T_{1} \nu, \int_{t_{n}}^{T} \rho(x(t)) d t \geqslant-T_{1} \nu$. Из
приведенных оценок следует, что

$$
\begin{aligned}
\int_{0}^{T} \rho(x(t)) d t & =\int_{0}^{t_{0}} \rho(x(t)) d t+\int_{t_{0}}^{t_{n}} \rho(x(t)) d t+\int_{t_{n}}^{T} \rho(x(t)) d t \\
& \geqslant-T_{1} \nu+k+2 T_{1} \nu-T_{1} \nu=k
\end{aligned}
$$

Второй случай $\left(n \leqslant n_{0}\right)$. В этом случае время, проводимое траекторией вне окрестностей $V_{j}$, не превосходит $(n+2) T_{1} \leqslant\left(n_{0}+2\right) T_{1}$; следовательно, время, проводимое внутри окрестности $V_{j}$, больше чем $T-\left(n_{0}+2\right) T_{1}$ и потому

$$
\int_{0}^{T} \rho(x(t)) d t \geqslant\left(T-\left(n_{0}+2\right) T_{1}\right) \theta-\left(n_{0}+2\right) T_{1} \nu>k .
$$

Отсюда следует справедливость леммы.

Закончим вывод условия $\left(\mathrm{H}^{*} \Lambda\right)$ из условия $(\mathrm{H} \Lambda)$.

В силу равенства (11) из условия $\operatorname{div} X(x)<0$ следует, что $\lambda(x)>\rho(x)$, и потому сушествуют такие $k>0, T>0$, что $\int_{0}^{T} \lambda(x(t)) d t>k$ для любого отрезка траектории длины $T$. Как мы видели выше, это неравенство вместе с доказанным ранее эквивалентно условию $\left(\mathrm{H}^{*} \Lambda\right)$. 
4.4. Вывод условия $\left(\mathrm{H}^{*} \mathrm{U}\right)$ из $(\mathrm{HU})$. Пусть на $P$ определено непрерывное и инвариантное относительно $d F_{P}$ семейство конусов $\mathscr{K}_{x}^{u}$, для которого выполнены условия а) и bu) из (HU). Как мы видели выше (лемма 2.7), при $\operatorname{div} X(x)<0$ отсюда следует выполнение условия bs) из (HU).

Мы приведем доказательство выполнения условия $\left(\mathrm{H}^{*} \mathrm{U}\right)$ с некоторыми пробелами, которые нетрудно восполнить.

Определим семейство конусов $\mathscr{K}_{x}^{u *}$ для точек $x \in P$ как множество таких векторов $q \in T_{x}^{*} U$, что $q$ ортогонален вектору $X(x)$ и одному из векторов $v \in \mathscr{K}_{x}^{u}$.

Для каж дой неподвижной точки $x_{j}$ типа $(L)$ построим окрестность $V_{j}$, как при доказательстве леммы 2.3. Нам потребуются следующие свойства окрестности $V_{j}$ : всякая положительная полутраектория, выходящая из $V_{j}$, пересекается с поверхностью $P$ во внутренней точке, а отрезок этой полутраектории от выхода из $V_{j}$ до пересечения с $P$ лежит вне всех окрестностей $V_{j}$.

Определим конус $\widetilde{\mathscr{K}}_{x}^{u *}$ в окрестности $V_{j}$ неподвижной точки $x_{j}$ типа $(L)$. Пусть $\lambda_{1}$ - минимальное собственное число матрицы $D\left(x_{j}\right), q_{1}$ - соответствующий левьй собственный вектор. Определим в $V_{j}$ конус $\mathscr{K}_{x}^{u *}$ как множество таких векторов $q$, что $\angle\left(q, q_{1}\right) \leqslant \alpha$, где $\alpha$ - достаточно малое положительное число. Будем предполагать, что окрестность $V_{j}$ достаточно мала.

Пусть $x(t)$ - траектория, $x(0) \in P, t_{1}$ - такое число, что либо $x\left(t_{1}\right) \in P$, либо $x\left(t_{1}\right) \in \cos \left(V_{j}\right)$ для некоторого $j$, причем $x(t)$ при $t \in\left(0, t_{1}\right)$ не принадлежит $P$ и не принадлежит никакой окрестности $V_{j}$. Для точек $x\left(t_{0}\right), t_{0} \in\left(0, t_{1}\right)$, определим конус $\mathscr{K}_{x\left(t_{0}\right)}^{u *}$ как множество таких векторов $q\left(t_{0}\right)$, что $q(t)$ - решение сопряженного уравнения в вариациях вдоль $x(t), q(0) \in \mathscr{K}_{x(0)}^{u *}$. В силу леммы 4.4 если окрестность $V_{j}$ достаточно мала, $t_{0}>t_{1}, x\left(t_{0}\right) \in V_{j}$, то $\mathscr{K}_{x\left(t_{0}\right)}^{u *} \subset \widetilde{\mathscr{K}}_{x\left(t_{0}\right)}^{u *}$.

Для полутраекторий $x(t), x(0) \in V_{j}$, определим конус $\mathscr{K}_{x\left(t_{0}\right)}^{u *}$ следующим образом. Пусть $t_{1}$ - момент выхода полутраектории из $V_{j}, t_{2}$ - точка пересечения полутраектории с поверхностью $P$. Построим конусы $\mathscr{K}_{x\left(t_{0}\right)}^{u *}$ для точек полутраектории $x(t)$ как множество таких векторов $q\left(t_{0}\right)$, что $q(t)$ - решение сопряженного уравнения в вариациях вдоль $x(t), q\left(t_{1}\right) \in \mathscr{K}_{x\left(t_{1}\right)}^{u *}$.

Таким образом, система конусов $\mathscr{K}_{x\left(t_{0}\right)}^{u *}$ построена для некоторой окрестности множества $\Lambda$.

Докажем инвариантность построенной системы конусов. Вопросы могут возникнуть только для точек $x \in P$. Пусть $x(t)$-полутраектория, $x(0) \in P, x\left(t_{1}\right) \in P$, и пусть $x(t) \notin P, x(t) \notin V_{j}$ для $t \in\left(0, t_{1}\right)$. Пусть $v(t), q(t)$ - решения уравнения в вариациях и сопряженного уравнения в вариациях вдоль $x(t), v(0) \in \mathscr{K}_{x(0)}^{u}$, $q(0) \in \mathscr{K}_{x(0)}^{u *}$, причем $v(0), q(0)$ ортогональны. Тогда в силу леммы 3.1 векторы $v\left(t_{1}\right), q\left(t_{1}\right)$ тоже ортогональны. Вектор $\widetilde{v}_{1}=d F v(0)$ принадлежит $\mathscr{K}_{x\left(t_{1}\right)}^{u}$ в силу инвариантности семейства $\mathscr{K}_{x}^{u}$ относительно $d F, v\left(t_{1}\right)$ принадлежит двумерному пространству, порож денному векторами $X\left(x\left(t_{1}\right)\right)$ и $\widetilde{v}_{1}$. Следовательно, $q\left(t_{1}\right)$ ортогонален векторам $X\left(x\left(t_{1}\right)\right)$ и $\widetilde{v}_{1}$ и поэтому принадлежит конусу $\mathscr{K}_{x\left(t_{0}\right)}^{u *}$.

Таким образом, инвариантное семейство конусов построено. Для этого семейства не имеют места непрерывность и инвариантность в том смысле, что

$$
\operatorname{clos}\left(\mathscr{K}_{x(0)}^{u *} d \Phi_{t}^{*}\right) \subset \operatorname{int}\left(\mathscr{K}_{x(t)}^{u *}\right),
$$

однако его можно модифицировать так, что соответствуюшее свойство будет иметь место, как и непрерьвность семейства. 
Приведем схему доказательства свойства b) из условия (H*U). Сначала определим функции $\widetilde{\lambda}(x, q), \widetilde{\rho}(x, q), q \in \mathscr{K}_{x}^{u *}$, следующим образом.

Пусть $x(t)$ - траектория, $q(t)$ - решение сопряженного уравнения в вариациях вдоль траектории $x(t), v_{1}(t), v_{2}(t)$ - два линейно независимых решения уравнения в вариациях вдоль траектории $x(t)$, причем $v_{1}(0), v_{2}(0)$ ортогональны некоторому вектору $q(t)$. Определим $\widetilde{\lambda}(x, q), \widetilde{\rho}(x, q)$ так, что

$$
\frac{d|q(t)|}{d t}=\widetilde{\lambda}(x(t), q(t))|q(t)|, \quad \frac{d S\left(v_{1}(t), v_{2}(t)\right)}{d t}=\widetilde{\rho}(x(t), q(t)) S\left(v_{1}(t), v_{2}(t)\right),
$$

где $S\left(v_{1}, v_{2}\right)$ - плошадь параллелограмма, порожденного векторами $v_{1}, v_{2}$.

Для функций $\widetilde{\lambda}(x, q), \widetilde{\rho}(x, q)$ выполнены свойства, аналогичные свойствам функций $\lambda(x), \rho(x)$, определенных ранее. В частности, справедливо равенство

$$
\widetilde{\lambda}(x, q)+\operatorname{div} X(x)=\widetilde{\rho}(x, q) .
$$

Далее мы докажем неравенство $\int_{0}^{T} \widetilde{\rho}(x(t), q(t)) d t>k$ для некоторых $k>0$, $T>0$ так же, как доказывали аналогичное неравенство для функции $\rho(x)$. Из последнего неравенства и из (17) следует, что $\int_{0}^{T} \widetilde{\lambda}(x(t), q(t)) d t>k$ для тех же констант $k, T$.

Свойство $\left(\mathrm{H}^{*} \mathrm{U}\right)$ доказано.

\section{$\S 5$. Неавтономные возмущения}

Как было сказано в начале статьи, неавтономным возмушением системы вида $\dot{x}=X(x)$ является система

$$
\dot{x}=Y(x, t)=X(x)+h(x, t),
$$

где $h(x, t)$ - малая функция. Систему $\dot{x}=X(x)$ мы будем называть невозмущенной, систему $\dot{x}=Y(x, t)$ - возмущенной. Мы будем рассматривать малость возмущения относительно нормы

$$
\|h\|=\sup _{x \in U, t \in \mathbb{R}^{1}, i, j \in\{1,2,3\}}\left(|h(x, t)|+\left|\frac{\partial\left(h_{i}(x, t)\right)}{\partial x_{j}}\right|+\left|\frac{\partial\left(h_{i}(x, t)\right)}{\partial t}\right|\right) .
$$

При этом частные производные второго порядка считаются ограниченньми.

Ранее для систем типа системы Лоренца была построена инвариантная окрестность $U$ множества $\Lambda$. Примем без доказательства тот факт, что можно построить новую окрестность с кусочно гладкой границей, в которую все траектории входят под ненулевьм углом. Далее мы будем считать, что $U$ - именно такая окрестность и что система вне окрестности $U$ не определена.

В этом случае если $h(x, t)$ достаточно мала, то вектор $Y(x, t)$ направлен тоже внутрь области $U$, и если $x(t)$ - решение системы $\dot{x}=Y(x, t), x\left(t_{0}\right) \in U$ при некотором $t_{0}$, то $x(t) \in U$ при любом $t>t_{0}$.

Мы будем рассматривать систему в расширенном фазовом пространстве. В нашем случае расширенное фазовое пространство - это прямое произведение $U \times \mathbb{R}^{1}$. 
Множество $U \times\{t\}, t \in \mathbb{R}^{1}$, обозначается через $U_{t}$. Касательное пространство к $U_{t}$ обозначается через $T_{x, t} U_{t}$. Пространство, сопряженное к пространству $T_{x, t} U_{t}$, обозначается через $T_{x, t}^{*} U_{t}$.

Мы будем допускать некоторую вольность, обозначая одним и тем же символом координаты точки $x=\left(x_{1}, x_{2}, x_{3}\right)$ в $U$ и в множествах $U_{t}$. Эта вольность оправдьвается тем, что все множества $U_{t}$ можно отождествить, поскольку система рассматривается в прямом произведении. Аналогично, решение $x(t)$ системы (19) может рассматриваться как лежащее в $U$ при каждом $t$, так и лежащее в $U_{t}$.

Вместо потока для возмушенной системы рассматривается семейство отображений $\widehat{\Phi}_{t_{1}, t_{2}}: U_{t_{1}} \rightarrow U_{t_{2}}$, которое определяется следуюшим образом. Пусть $x(t)-$ решение системы, $x\left(t_{1}\right)=x$. Тогда $\widehat{\Phi}_{t_{1}, t_{2}}(x)=x\left(t_{2}\right)$. Очевидно, что для невозмущенной системы $\widehat{\Phi}_{t_{1}, t_{2}}=\Phi_{t_{2}-t_{1}}$.

Расслоение $U \times \mathbb{R}^{1}$ на множества $U_{t}$ инвариантно относительно $\widehat{\Phi}_{t_{1}, t_{2}}$. Поэтому $d \widehat{\Phi}_{t_{1}, t_{2}}$ переводит вектор, касательньй к множеству $U_{t_{1}}$, в вектор, касательный к множеству $U_{t_{2}}$.

A ттрактором мы будем называть множество $\Lambda \subset U \times \mathbb{R}^{1}$, состоящее из таких точек $\left(x_{0}, t_{0}\right)$, что решение $x(t)$ принадлежит $U_{t}$ при всех $t \in \mathbb{R}^{1}$, если $x\left(t_{0}\right)=x_{0}$. Через $\Lambda_{t}$ обозначается множество $\Lambda \cap U_{t}$.

5.1. Уравнение в вариация х и сопряженное уравнение в вариация $x$ для неавтономного возмущения. Пусть $D(x, t)-(3 \times 3)$-матрица из частных производных $\partial Y_{i}(x, t) / \partial x_{j}$. Уравнение в вариациях, соответствуюшее решению $x(t)$ возмушенной системы, имеет вид $\dot{v}=D(x(t), t) v$, сопряженное уравнение в вариациях имеет вид $\dot{q}=-q D(x(t), t)$. При этом считается, что при каждом $t \in \mathbb{R}^{1}$ вектор $v(t)$ - элемент пространства $T_{(x(t), t)} U_{t}$, а вектор $q(t)$ - элемент пространства $T_{(x(t), t)}^{*} U_{t}$.

Отметим, что для неавтономного возмушения системы имеет место сохранение скалярного произведения (остается справедливой лемма 3.1). Доказательство этой леммы в точности повторяет доказательство для автономного случая.

Сформулируем определение сингулярно гиперболической структуры для неавтономного возмушения.

УСловие $\left(\mathrm{H}^{*} \mathrm{UN}\right)$. Для каждой точки $x \in U_{t}$ определен конус $\mathscr{K}_{x, t}^{u *} \subset T_{x, t}^{*} U_{t}$. Семейство конусов $\mathscr{K}_{x, t}^{u *}$ инвариантно в том смысле, что сушествует $t_{1}>0$ такое, что если решение $x(t) \in U_{t}$ при $t \geqslant t_{0}, q(t)$ - решение сопряженного уравнения в вариациях вдоль $x(t), q\left(t_{0}\right) \in \cos \mathscr{K}_{x\left(t_{0}\right), t_{0}}^{u *}$, то $q(t) \in \operatorname{int} \mathscr{K}_{x, t}^{u *}$ при всех $t>t_{0}+t_{1}$. Кроме того, существуют константы $c_{1}>0, \gamma_{1}>0, c_{2}>0, \gamma_{2}>0$ такие, что

a) если $q(t)$ - решение сопряженного уравнения в вариациях вдоль решения $x(t), q\left(t_{0}\right) \in \mathscr{K}_{x\left(t_{0}\right), t_{0}}^{u *}, t>t_{0}$, то $|q(t)|>c_{1} e^{\gamma_{1}\left(t-t_{0}\right)}\left|q\left(t_{0}\right)\right| ;$

b) если $v_{1}(t), v_{2}(t)$ - решения уравнения в вариациях, ортогональные некоторому решению $q(t)$ сопряженного уравнения в вариациях, такому, что $q\left(t_{0}\right) \in \mathscr{K}_{x\left(t_{0}\right), t_{0}}^{u *}$, то при всех $t>t_{0}$ имеет место

$$
S\left(v_{1}(t), v_{2}(t)\right)>c_{2} e^{\gamma_{2}\left(t-t_{0}\right)} S\left(v_{1}\left(t_{0}\right), v_{2}\left(t_{0}\right)\right),
$$

где $S\left(v_{1}(t), v_{2}(t)\right)$ - плошадь параллелограмма, порожденного векторами $v_{1}(t), v_{2}(t)$. 
Как и в автономном случае, для каждой точки $x \in \Lambda_{t}$ определим одномерное подпространство $E_{x, t}^{u *} \subset T_{x(t)}^{*} U_{t}$. Семейство пространств $E_{x, t}^{u *}$ инвариантно. Это означает, что если $x(t)$ - траектория, $x\left(t_{1}\right) \in \Lambda_{t_{1}}$ при каком-то $t_{1}, q(t)$ - решение сопряженного уравнения в вариациях, $q\left(t_{1}\right) \in E_{x\left(t_{1}\right), t_{1}}^{u *}$, то $q(t) \in E_{x(t), t}^{u *}$ при всех $t$. Семейство пространств $E_{x, t}^{u *}$ зависит от $x$ и $t$ непрерывно на множестве $\Lambda=\bigcup_{t} \Lambda_{t}$ и дифференцируемо вдоль решений.

Справедлива лемма 3.3 , согласно которой пространство $E_{x, t}^{u *}$ ортогонально вектору скорости $Y(x, t)$. Доказательство точно такое же, как и для автономного случая.

Аттрактор $\Lambda$ мы будем называть ориентируемым, если существует векторное поле $p(x, t) \in E_{x, t}^{u *}$, непрерывно зависяшее от $x, t$ и дифференцируемое вдоль траекторий.

5.2. Грубые и негрубые свойства. Мы будем называть свойство невозмущенной системы грубым, если оно сохраняется для малых неавтономных возмущений. Свойство называется негрубым, если найдется сколь угодно малое неавтономное возмущение, для которого соответствующее свойство не имеет места.

Приведем без доказательства несколько простых утверждений о грубых и негрубых свойствах.

Наличие гиперболической неподвижной точки является грубьм свойством. Пусть $x_{0}$ - неподвижная точка невозмущенной системы. Тогда у возмущенной системы в окрестности точки $x_{0}$ имеется единственное решение $x_{0}(t)$, определенное при любом $t \in \mathbb{R}^{1}$ и такое, что в каждой точке $x_{0}(t)$ имеется разложение пространства $\mathbb{R}^{3}$ в прямую сумму подпространств $\mathbb{R}^{3}=E_{x, t}^{u} \oplus E_{x, t}^{s}$; если $v(t)$ - решение уравнения в вариациях, $v\left(t_{1}\right) \in E_{x\left(t_{1}\right), t_{1}}^{u}$, то $|v(t)|$ экспоненциально растет при $t \rightarrow+\infty$; если же $v\left(t_{1}\right) \in E_{x\left(t_{1}\right), t_{1}}^{s}$, то $|v(t)|$ экспоненциально растет при $t \rightarrow-\infty$. Если возмушение периодично, то и траектория $x(t)$, и разложение $\mathbb{R}^{3}=E_{x, t}^{u} \oplus E_{x, t}^{s}$ периодичны с тем же периодом.

Гиперболическая структура в окрестности гиперболической периодической траектории не является грубым свойством. Рассмотрим периодическое возмущение периода $T$. Его можно привести стандартньм образом к системе порядка 4 с правой частью, не зависящей от $t$. Пусть $\varphi$ - угловая переменная на окружности, $\beta=2 \pi / T$. Заменим невозмушенную систему на систему

$$
\left\{\begin{array}{l}
\dot{x}=X(x)+h(x, \varphi) \\
\dot{\varphi}=\beta
\end{array}\right.
$$

в пространстве $U \times S^{1}$. Отображение последования $\widetilde{\Phi}$ на трехмерной поверхности $U \times\{0\}$ является возмущением отображения $\Phi_{T}$.

Рассмотрим периодическую траекторию невозмушенной системы с двумерными устойчивьм и неустойчивым многообразиями. Она является окружностью, инвариантной относительно $\Phi_{T}$ и гиперболичной. Отображение $\widetilde{\Phi}$ является малым возмущением отображения $\Phi_{T}$. При малых возмущениях инвариантная окружность сохраняется и на ней появляется последовательность чередующихся устойчивых и неустойчивых траекторий (относительно сужения отображения $\widetilde{\Phi}$ на эту 
окружность). Устойчивая периодическая точка на самом деле является гиперболической (если ее рассматривать в окрестности $U$ ) с одномерным неустойчивым многообразием. Неустойчивая является гиперболической с двумерным неустойчивым многообразием. Таким образом, в окрестности цикла невозмущенной системы появляются гиперболические циклы как с одномерньм, так и с двумерньм неустойчивым многообразием. Это говорит о разрушении гиперболической структуры при малом неавтономном возмущении.

Наличие сингулярно гиперболической структуры является грубым свойством. Для невозмущенной системы конусы $\mathscr{K}_{x, t}^{u *}$ не зависят от $t$.

Пусть $\mathscr{K}_{x, t}^{u *}$ - конусы, $c_{1}, \gamma_{1}, c_{2}, \gamma_{2}$ - константы для невозмущенной системы, фигурирующие в условии $\left(\mathrm{H}^{*} \mathrm{U}\right)$. В качестве конусов можно взять те же самые конусы для возмущенной системы. Нетрудно видеть, что если возмушение достаточно мало, то инвариантность конусов сохраняется. Константы $c_{1}, \gamma_{1}, c_{2}, \gamma_{2}$ для возмущенной системы несколько изменятся, но останутся положительными. Поэтому наличие сингулярно гиперболической структуры является грубым свойством.

5.3. Отсутствие устойчивых решений. Докажем следуюшее утверждение.

ТЕОРема 5.1. Пусть система, определяемая уравнением $\dot{x}=Y(x, t)$, сингулярно гиперболична. Тогда устойчивъе траектории отсутствуют.

ДоказАТЕЛЬСтво. Для заданного решения $x(t), t \geqslant t_{0}$, определим решение сопряженного уравнения в вариациях $q(t), q\left(t_{0}\right) \in \mathscr{K}_{x\left(t_{0}\right), t_{0}}^{u *}$. Тогда в силу инвариантности системы конусов $q(t) \in \mathscr{K}_{x(t), t}^{u *}$ при любом $t>t_{0}$. Определим плошадку $\Pi_{x(t), t} \in T_{x(t), t} U$ как множество векторов $v \in T_{x(t), t} U$, ортогональных вектору $q(t)$. Из условия (H* $\mathrm{UN})$ следует, что $S\left(v_{1}(t), v_{2}(t)\right)$ экспоненциально растет. В качестве вектора $v_{1}$ рассмотрим вектор $Y(x, t)$. Вектор $Y(x, t)$ ограничен. Следовательно, $\left|v_{2}(t)\right|$ экспоненциально растет. Таким образом, уравнение в вариациях вдоль любой траектории $x(t)$ имеет экспоненциально растущее решение. Из равномерной ограниченности производных второго порядка следует, что решение $x(t)$ неустойчиво. Теорема доказана.

\section{§6. Обобщение на случай произвольной размерности}

6.1. Определение сингулярно гиперболических систем. Мы сформулируем определение сингулярно гиперболической системы в пространстве $\mathbb{R}^{n}$. Приведенное ниже определение без труда переносится на случай системы, заданной на произвольном римановом многообразии.

Определение будет формулироваться для неавтономной системы. Сохраняются все обозначения и определения предыдушего параграффа.

Для пространства $\mathbb{R}^{n}$ очевидны обобшения определения матрицы $D(x, t)$, уравнения в вариациях, сопряженного уравнения в вариациях и т.д. Остается верньм сохранение скалярного произведения (лемма 3.1).

Предполагается, что в ограниченной области $U \subset \mathbb{R}^{n}$ определено $C^{1}$-гладкое векторное поле $Y(x, t)$. Вне $U$ поле $Y(x, t)$ не определено. Обозначим через $\Lambda \subset$ $U \times \mathbb{R}^{1}$ множество таких точек $\left(x_{0}, t_{0}\right) \in U \times \mathbb{R}^{1}$, что решение $x(t)$ уравнения $\dot{x}=Y(x, t)$ с начальным условием $x\left(t_{0}\right)=x_{0}$ определено (и, следовательно, принадлежит $U)$ при всех $t \in \mathbb{R}^{1}$. 
Множество $\Lambda$ называется локально максимальным множсеством.

Если положительная полутраектория $x(t)$ всякой точки $\left(x_{0}, t_{0}\right) \in U \times \mathbb{R}^{1}$ лежит в $U$, то $\Lambda$ - аттрактор. Зафиксируем два натуральных числа $m, k, m \leqslant n-2$, $2 \leqslant k \leqslant n-m$.

ОПРЕДЕЛЕНИЕ 6.1. Система, определяемая в $U \subset \mathbb{R}^{n}$ уравнением $\dot{x}=Y(x, t)$, называется сингулярно гиперболической, если выполнено следуюшее.

УСловиЕ $\left(\mathrm{H}_{n}^{*} \mathrm{U}\right)$. Для каждой точки $(x, t) \in U \times \mathbb{R}^{1}$ определен конус $\mathscr{K}_{x, t}^{u *} \subset$ $T_{x, t}^{*} U$ с $m$-мерньм осевым пространством. Семейство конусов $\mathscr{K}_{x, t}^{u *}$ непрерьвно зависит от точки $(x, t) \in U \times \mathbb{R}^{1}$, инвариантно, т.е. сушествует такое $t_{1}>0$, что если $x(t)$ - решение, $x(t) \in U$ при $t \in\left[t_{0}, t_{0}+t_{1}\right], q(t)$ - решение сопряженного уравнения в вариациях вдоль $x(t), q\left(t_{0}\right) \in \operatorname{clos} \mathscr{K}_{\left(x\left(t_{0}\right), t_{0}\right)}^{u *}$, то $q(t) \in \operatorname{int} \mathscr{K}_{(x(t), t)}^{u *}$ при всех $t>t_{0}+t_{1}$. Кроме того, сушествуют такие константы $c_{1}>0, \gamma_{1}>0$, $c_{2}>0, \gamma_{2}>0$, что

а) если $x(t)$ - решение, определенное при $t \in(\alpha, \beta), q(t)$ - решение сопряженного уравнения в вариациях вдоль $x(t), q(t) \in \mathscr{K}_{(x(t), t)}^{u *}, \alpha<t_{0}<t<\beta$, то $|q(t)|>c_{1} e^{\gamma_{1}\left(t-t_{0}\right)}\left|q\left(t_{0}\right)\right|$

b) если $x(t)$ - решение, определенное при $t \in(\alpha, \beta), q(t)$ - решение сопряженного уравнения в вариациях вдоль $x(t), q(t) \in \mathscr{K}_{(x(t), t)}^{u *}, v_{1}(t), \ldots, v_{k}(t)-$ решения уравнения в вариациях вдоль $x(t)$, ортогональные $q(t), \alpha<t_{0}<$ $t<\beta$, то

$$
V_{k}\left(v_{1}(t), \ldots, v_{k}(t)\right)>c_{2} e^{\gamma_{2}\left(t-t_{0}\right)} V_{k}\left(v_{1}\left(t_{0}\right), \ldots, v_{k}\left(t_{0}\right)\right)
$$

где $V_{k}\left(v_{1}(t), \ldots, v_{k}(t)\right)-k$-мерный объем параллелепипеда, порожденного векторами $v_{1}(t), \ldots, v_{k}(t)$.

6.2. Неподвижные точки. Имеет смысл рассматривать неподвижные точки только для автономных систем.

Пусть в области $U \in \mathbb{R}^{n}$ определена автономная система $\dot{x}=X(x)$, удовлетворяющая условию $\left(\mathrm{H}_{n}^{*} \mathrm{U}\right), x_{0} \in U-$ неподвижная точка. Опишем возможный спектр матрицы $D\left(x_{0}\right)$ (аналог леммы 3.4$)$.

Оказывается, что сушествует такое $\theta>0$, что спектр матрицы $D\left(x_{0}\right)$ делится на три части. Первая часть состоит из таких собственных значений $\lambda$, что $\operatorname{Re} \lambda<-\theta$. Этим собственным значениям соответствует подпространство $E_{x_{0}}^{s}$ пространства $T_{x_{0}} U, \operatorname{dim} E_{x_{0}}^{s}=m$. Вторая часть состоит из таких собственных значений $\lambda$, что $-\theta<\operatorname{Re} \lambda \leqslant 0$. Этим собственньм значениям соответствует подпространство $E_{x_{0}}^{0}$ пространства $T_{x_{0}} U, \operatorname{dim} E_{x_{0}}^{0} \leqslant k-1$. Третья часть состоит из таких собственных значений $\lambda$, что $\operatorname{Re} \lambda>0$. Этим собственным значениям соответствует подпространство $E_{x_{0}}^{u}$ пространства $T_{x_{0}} U, \operatorname{dim} E_{x_{0}}^{u} \leqslant n-m$. Следовательно, пространство $T_{x_{0}} U$ разлагается в прямую сумму $T_{x_{0}} U=E_{x_{0}}^{s} \oplus E_{x_{0}}^{0} \oplus E_{x_{0}}^{u}$.

Для любых $k$ собственных значений $\lambda_{1}, \ldots, \lambda_{k}$, соответствующих собственным векторам из пространства $E^{0} \oplus E^{u}$, имеет место неравенство

$$
\operatorname{Re}\left(\lambda_{1}+\cdots+\lambda_{k}\right)>0
$$


Кратные числа в этой сумме должны встречаться в количестве, не превышаюшем кратности соответствующего собственного числа.

Если $\operatorname{dim} E^{0}=0$, то в некоторой окрестности $U_{x_{0}}$ точки $x_{0}$ нет неблуждающих точек, кроме самой точки $x_{0}$, а множество $\Lambda \cap U_{x_{0}}$ состоит из точки $x_{0}$ и ее неустойчивого многообразия.

Пространство $E_{x_{0}}^{u *}$ является ортогональньм дополнением к $E^{0} \oplus E^{u}$.

Доказательство этих утверждений принципиально не отличается от доказательства леммы 3.4 .

6.3. Соотношение с классическим определением гиперболического множества. Классическое определение гиперболического множества $\Lambda$ приведено в определении 2.6 .

Если система автономная, неподвижные точки отсутствуют, вьполнено условие $\left(\mathrm{H}_{n}^{*} \mathrm{U}\right)$ и $k=2$, то поток, порожденньй уравнением, гиперболичен на $\Lambda$ в смысле классического определения.

Обратно, если система гиперболична на инвариантном множестве $\Lambda$ в смысле классического определения, то существует такая окрестность $U_{1}$ множества $\Lambda$, на которой выполнено условие $\left(\mathrm{H}_{n}^{*} \mathrm{U}\right)$ с $k=2$.

Пусть автономная система $\dot{x}=X(x)$ удовлетворяет условию $\left(\mathrm{H}_{n}^{*} \mathrm{U}\right)$. Обозначим через $U^{+}$множество таких точек $x \in U$, что положительная полутраектория точки $x$ целиком лежит в $U$. Если $\Lambda$ - аттрактор, то $U^{+}=U$.

Оказывается, что на $U^{+}$существует семейство инвариантных устойчивых подпространств $E_{x}^{s} \subset T_{x} U$. Пространства $E_{x}^{s}$ зависят от точки $x$ непрерывно, семейство $E_{x}^{s}$ дифференцируемо вдоль траекторий. Размерность пространств $E_{x}^{s}$ равна $m$. Кроме того, если $x(t)$ - решение, $x(t) \in U^{+}, v(t)$ - решение уравнения в вариациях, $v(t) \in E_{x(t)}^{s}$, то $|v(t)|<c_{3} e^{-\gamma_{1} t}|v(0)|$, где константа $\gamma_{1}$ определена в условии $\left(\mathrm{H}_{n}^{*} \mathrm{U}\right), c_{3}>0$.

Кроме того, для точек $x \in U^{+}$существует семейство локальных устойчивых многообразий, инвариантное относительно потока.

Таким образом, в пространстве $\mathbb{R}^{3}$ для системы типа системы Лоренца в построенной окрестности существует инвариантное семейство локальных устойчивых многообразий.

6.4. Комментарии. В основе идеи введения сингулярной гиперболичности, как мы видели, лежат две идеи: переход к сопряженному уравнению в вариациях и свойство роста площадей в некоторых двумерных направлениях касательного пространства. Эти идеи не новы. Можно отметить работу [21], в которой использовались даже более обшие преобразования дифференциальных форм (можно заметить, что элемент пространства, сопряженного касательному, - это дифференциальная 1-форма). В работе [16] одно из условий формулировалось в виде роста площади вдоль неустойчивой сепаратрисы неподвижной точки. Доказательство существования инвариантного семейства двумерных подпространств вдоль сепаратрисы по сушеству использовало тот факт, что семейство конусов $\mathscr{K}_{x}^{u *}$ в окрестности неподвижной точки является инвариантным. Поэтому существует инвариантное семейство подпространств, которые в настоящей работе обозначены $E_{x}^{u *}$, определенные для точек $x$, принадлежащих неустойчивому многообразию неподвижной точки. 
Как говорилось вьшше, в работе [22] формулировалось условие, наиболее близкое к нашему условию сингулярной гиперболичности, так называемая псевдо-гиперболичность. Это означает, что имеется строго устойчивое одномерное слоение, а в двумерном направлении, трансверсальном этому слоению, имеет место экспоненциальный рост площадей.

В работах [22], [23] условие роста площадей в двумерных направлениях использовался для доказательства ряда утверж дений, простейший вариант которых можно сформулировать следующим образом.

Предположим, что диффеоморфизм на трехмерном многообразии имеет неподвижную точку, собственные числа дифференциала диффеоморфизма в неподвижной точке таковы, что $\left|\lambda_{1}\right|<\left|\lambda_{2}\right|<1<\left|\lambda_{3}\right|,\left|\lambda_{2} \lambda_{3}\right|>1$, и имеется общая точка устойчивого и неустойчивого многообразий (т.е. имеется гомоклиническая траектория). Тогда имеется окрестность гомоклинической траектории, в которой достаточно малое (в $C^{1}$-топологии) возмушение исходного диффеоморфизма не имеет устойчивых периодических траекторий.

Прямое использование этого утверждения позволило бы доказать следуюшее утверждение, которое является частньм случаем приведенного в настояшей работе.

Предположим, что значения параметров таковы, что у потока системы Лоренца неустойчивая сепаратриса неподвижной точки $O(0,0,0)$ лежит в устойчивом многообразии этой точки. Тогда имеется окрестность сепаратрисы, в которой малое периодическое возмушение не имеет устойчивых периодических траекторий.

Однако, насколько мне известно, в литературе не встречалась формулировка условия гиперболичности, повторяюшая условие сингулярной гиперболичности в приведенном в настояшей работе виде.

В заключение автор с удовольствием выражает благодарность Р. В. Плыкину, Л. П. Шильникову и С.В. Гонченко за полезные обсуждения.

\section{Список литературы}

1. Saltzman B. Finite amplitude free convectiions as an initial value problem. I // J. Atmospheric Sci. 1962. V. 19. № 2. P. 329-341.

2. Lorenz E. N. Deterministic nonperiodic flow // J. Atmospheric Sci. 1963. V. 20. № 2 . P. 130-141.

3. McLaughlin B., Martin P. C. Transition to turbulence of statically stressed fluids // Phys. Rev. Lett. 1974. V. 33. № 1. P. 1189-1192.

4. Yorke J. A., Yorke E. D. Metastable chaos: The transition to sustained chaotic oscillations in a model of Lorenz // J. Statist. Phys. 1979. V. 21. P. 263-277.

5. Guckenheimer J., Williams R. F., Structural stability of Lorenz attractors // Inst. Hautes Études Sci. Publ. Math. 1979. V. 50. P. 59-72.

6. Rand D. The topological classification of Lorenz attractors // Math. Proc. Cambridge Philos. Soc. 1978. V. 83. № 3. P. 451-460.

7. Афраймович В. С., Быков В. В., Шильников Л. П. О притягивающих негрубых предельных множествах типа аттрактора Лоренца // Труды ММО. 1982. Т. 44. С. 150-212.

8. Šlačkov S. V. Pseudo-orbit tracing property and structural stability of expanding maps of the interval // Ergodic Theory Dynam. Systems. 1992. V. 12. P. 573-587.

9. Sparrow $C$. The Lorenz attractor: Bifurcations, chaos and strange attractors. New York: Springer-Verlag, 1982.

10. Бунимович Л. А., Синай Я.Г. Стохастичность аттрактора в модели Лоренца // Нелинейные волны / ред. А.В. Гапонов-Грехов. М.: Наука, 1979. С. 212-226. 
11. Сатаев E. A. Инвариантные меры для гиперболических отображений с особенностями // УМН. 1992. Т. 47. № 1. С. 147-202.

12. Сатаев $E$. A. Гиббсовские меры для одномерных аттракторов гиперболических отображений с особенностями // Изв. РАН. Сер. матем. 1992. Т. 56. №6. С. 1328-1344.

13. Pesin Ya. B. Dynamical systems with generalised hyperbolic attractors: hyperbolic, ergodic and topological properties // Ergodic Theory Dynam. Systems. 1992. V. 12. P. 123-151.

14. Tucker $W$. A rigorous ODE solver and Smale's 14th problem // Found. Comput. Math. 2002. V. 2. P. 53-117.

15. Rychlik $M$. Lorenz attractor through Sil'nicov-type bifurcation. I // Ergodic Theory Dynam. Systems. 1990. V. 10. № 4. P. 793-821.

16. Robinson C. Homoclinic bifurcation to a transitive attractor of Lorenz type // Nonlinearity. 1989. V. 2. № 4. P. 495-518.

17. Плькин P. B., Сатаев E. А., Шлячков C. В. Странные аттракторы // Итоги науки и техники. Совр. проблемы матем. Фундамент. направления. Динамические системы. Т. 9. М.: ВИНИТИ, 1991. С. 72-144.

18. Sternberg Sh. On the structure of local homeomorphisms of Euclidean $n$-space. II // Amer. J. Math. 1958. V. 80. P. 623-631.

19. Chen K. T. Equivalence and decomposition of vector fields about an elementary critical point // Amer. J. Math. 1963. V. 85. P. 693-722.

20. Kelly A. The stable, center-stable, center, center-unstable, unstable manifolds // J. Differential Equations. 1967. V. 3. №4. P. 546-570.

21. Аносов Д. В. Геодезические потоки на замкнутых римановых многообразиях отрицательной кривизны // Труды МИАН. 1967. Т. 90. С. 1-210.

22. Гонченко С. В., Тураев Д. В., Шильников Л. П. О существовании областей Ньюхауса вблизи систем с негрубой гомоклинической кривой Пуанкаре (многомернњй случай) // Докл. РАН. 1993. Т. 329. № 4. С. 404-407.

23. Тураев Д. В., Шильников Л. П. Пример дикого странного аттрактора // Матем. сб. 1998. Т. 189. № 4. С. 137-160.

Обнинский государственный технический университет

Поступила в редакцию атомной энергетики 12.05.2004 и 01.12.2004

E-mail: sataev@iate.obninsk.ru 